\title{
NUMERICAL APPROXIMATIONS OF ALGEBRAIC RICCATI EQUATIONS FOR ABSTRACT SYSTEMS MODELLED BY ANALYTIC SEMIGROUPS, AND APPLICATIONS
}

\author{
I. LASIECKA AND R. TRIGGIANI
}

\begin{abstract}
This paper provides a numerical approximation theory of algebraic Riccati operator equations with unbounded coefficient operators $A$ and $B$, such as arise in the study of optimal quadratic cost problems over the time interval $[0, \infty]$ for the abstract dynamics $\dot{y}=A y+B u$. Here, $A$ is the generator of a strongly continuous analytic semigroup, and $B$ is an unbounded operator with any degree of unboundedness less than that of $A$. Convergence results are provided for the Riccati operators, as well as for all the other relevant quantities which enter into the dynamic optimization problem. The present numerical theory is the counterpart of a known continuous theory. Several examples of partial differential equations with boundary/point control, where all the required assumptions are verified, illustrate the theory. They include parabolic equations with $L_{2}$-Dirichlet control, as well as plate equations with a strong degree of damping and point control.
\end{abstract}

\section{INTRODUCTION: CONTINUOUS AND DISCRETE OPTIMAL CONTROL PROBLEMS; MAIN RESULTS; LITERATURE}

1.1. Statement of the continuous problem: Assumptions and main results. Consider the following optimal control problem: Given the dynamical system,

$$
y_{t}=A y+B u ; \quad y(0)=y_{0} \in H,
$$

minimize the quadratic functional

$$
J(u, y)=\int_{0}^{\infty}\left[\|R y(t)\|_{Z}^{2}+\|u(t)\|_{U}^{2}\right] d t
$$

over all $u \in L_{2}(0, \infty ; U)$, with $y$ a solution of (1.1) with control function $u$.

Received March 12, 1990; revised August 8, 1990.

1980 Mathematics Subject Classification (1985 Revision). Primary 65K99, 65P05.

This research was partially supported by the National Science Foundation under Grant DMS-8796320 and by the Air Force Office of Scientific Research under Grant AFOSR-87-0321. This paper was presented by the first-named author at the Workshop on Computational Aspects of Control held at the Center for the Mathematical Sciences, University of Wisconsin, May 1988.

Because of the paper's length, most of its technical proofs are given in the Supplement section of this issue. Should this hinder the reading, the original manuscript-which incorporates the proofs in the body of the paper in a consequential manner-is available by the authors upon request. 
We shall make the following assumptions on (1.1), (1.2):

(i) $H, U$, and $Z$ are Hilbert spaces.

(ii) $A$ : $H \supset \mathscr{D}(A) \rightarrow H$ is the generator of a strongly continuous (s.c.) analytic semigroup $e^{A t}$ on $H, t>0$, generally unstable on $H$, i.e., with $\omega_{0}=\lim [(\ln \|\exp (A t)\|) / t]>0$ as $t \rightarrow+\infty$ in the uniform norm $\mathscr{L}(H)$, so that $\left\|e^{A t}\right\| \leq M e^{\left(\omega_{0}+\varepsilon\right) t}$ for all $\varepsilon>0, t \geq 0$, and $M$ depending on $\omega_{0}+\varepsilon$; we then consider throughout the translation $\widehat{A}=-A+\omega I, \omega=$ fixed $>\omega_{0}$, so that $\hat{A}$ has well-defined fractional powers on $H$ and $-\widehat{A}$ is the generator of an s.c. analytic semigroup $e^{-\widehat{A t}}$ on $H$ satisfying $\left\|e^{-\widehat{A t}}\right\| \leq \widehat{M} e^{-\hat{\omega} t}, t \geq$ $0 ; \hat{\omega}=\omega-\omega_{0}-\varepsilon>0$; it will be used without further explicit note that $[\mathscr{D}(\widehat{A}), H]_{1-\theta}=\mathscr{D}\left(\hat{A}^{\theta}\right), 0<\theta<1$, e.g., [28, Theorem 1.25.3, p. 103].

(iii) $B: U \supset \mathscr{D}(B) \rightarrow\left[\mathscr{D}\left(A^{*}\right)\right]^{\prime}$, the dual of $\mathscr{D}\left(A^{*}\right)$ with respect to the $H$ topology, $A^{*}$ being the $H$-adjoint of $A$; more precisely, it is assumed that $B^{*}$ is $\hat{A}^{* \gamma}$-bounded, or equivalently,

$$
(\widehat{A})^{-\gamma} B \in \mathscr{L}(U ; H) \text { for some constant } \gamma, 0 \leq \gamma<1 .
$$

(iv) The operator $R$ is bounded:

$$
R \in \mathscr{L}(H, Z) .
$$

Hypotheses (i)-(iv) are assumed to be in force throughout the paper and shall not be repeated.

The next assumption guarantees existence of a unique optimal pair $\left\{u^{0}, y^{0}\right\}$ of the optimal control problem (1.1), (1.2):

(v) Stabilizability Condition (S.C.):

$$
\left\{\begin{array}{l}
\text { there exists } F \in \mathscr{L}(H ; U) \text { such that the s.c. analytic semigroup } \\
e^{(A+B F) t} \text { (as guaranteed by }(1.3) \text {, see below) is exponentially } \\
\text { stable on } H \text {, i.e., }\left\|e^{(A+B F) t}\right\|_{\mathscr{L}(H)} \leq M_{F} e^{-\omega_{F} t} \text { for some } \omega_{F}>0 .
\end{array}\right.
$$

(Equation (1.3) says that $F^{*} B^{*}$ is $\left((\widehat{A})^{*}\right)^{\gamma}$-bounded; thus, since $\gamma<1, A^{*}+$ $F^{*} B^{*}$ is the generator of an s.c. analytic semigroup on $H$, and the same holds for $A+B F$.)

Finally, we shall make an assumption which guarantees uniqueness of the solution of the corresponding Algebraic Riccati Equation.

(vi) Detectability Condition (D.C.):

$$
\left\{\begin{array}{l}
\text { there exists } K \in \mathscr{L}(Z ; H) \text { such that the s.c. analytic } \\
\text { semigroup } e^{(A+K R) t} \text { is exponentially stable on } H, \text { i.e., } \\
\left\|e^{(A+K R) t}\right\|_{\mathscr{L}(H)} \leq M_{K} e^{-\omega_{K} t} \text { for some } \omega_{k}>0 .
\end{array}\right.
$$

The following main result for problem (1.1), (1.2) has been established in the literature either directly $[9,8]$ (from the Riccati equation to the control 
problem), or through a variational argument [17] (from the control problem to the Riccati equation).

Theorem 1.0 $[9,17,8]$. (1) Under the stabilizability condition $($ S.C. $)=(1.5)$, there is a unique solution $\left\{u^{0}, y^{0}\right\}$ of the optimal control problem (1.1), (1.2).

(2) Under the additional detectability condition $(D . C)=.(1.6)$, there is a unique nonnegative operator $P=P^{*} \in \mathscr{L}(H)$ such that, with $u^{0}(t)=u^{0}\left(t ; y_{0}\right)$ and $y^{0}(t)=y^{0}\left(t ; y_{0}\right), y_{0} \in H$, we have

$$
u^{0}(t)=-B^{*} P y^{0}(t), \quad 0<t<\infty,
$$

where $(B u, v)_{H}=\left(u, B^{*} v\right)_{U}$, and $P$ satisfies the following Algebraic Riccati Equation (A.R.E.):

$$
\begin{gathered}
\left(A^{*} P x, y\right)_{H}+(P A x, y)_{H}+\left(R^{*} R x, y\right)_{H}-\left(B^{*} P x, B^{*} P y\right)_{U} \\
=0 \quad \forall x, y \in \mathscr{D}\left(\widehat{A}^{\varepsilon}\right), \text { any } \varepsilon>0 .
\end{gathered}
$$

Moreover,

$$
\left(\widehat{A}^{*}\right)^{1-\varepsilon} P \in \mathscr{L}(H) \quad \forall \varepsilon>0
$$

( $\varepsilon=0$, if $A$ is self-adjoint or normal, or similar to a normal operator);

$$
J\left(u^{0}, y^{0}\right)=\left(P y_{0}, y_{0}\right)_{H}
$$

$$
B^{*} P \in \mathscr{L}(H, U) \text {; }
$$

(6) the s.c. analytic semigroup $\Phi(t)=e^{A_{P} t}=e^{\left(A-B B^{*} P\right) t}$ generated by $A_{P}=$ $A-B B^{*} P$ is exponentially stable,

$$
\left\|e^{\left(A-B B^{*} P\right) t}\right\|_{\mathscr{L}(H)} \leq M_{P} e^{-\omega_{P} t} \text { for some } \omega_{P}>0 .
$$

Further properties are collected in $\S 2.1$; see in particular identity (2.10) for $P$.

1.2. Approximation of dynamics and related properties. The main goal of this paper is to provide a numerical algorithm for the computation of the solution to the Algebraic Riccati Equation (A.R.E.) and to prove the desired convergence results.

\subsubsection{Approximation assumptions.}

Approximating subspaces. We introduce a family of approximating subspaces $V_{h} \subset H \cap \mathscr{D}\left(B^{*}\right)$, where $h$ is a parameter of discretization which tends to zero, $0<h \leq h_{0}$. Let $\Pi_{h}$ be the $H$-orthogonal projection of $H$ onto $V_{h}$ with the usual approximating property

$$
\left\|\Pi_{h} x-x\right\|_{H} \rightarrow 0 \text { for all } x \in H .
$$


Approximation of $A$. Let $A_{h}: V_{h} \rightarrow V_{h}$ be an approximation of $A$ which satisfies the following requirements (A.1) and (A.2):

(A.1) uniform analyticity; formulation in $t$-domain:

$$
\left\|A_{h}^{\theta} e^{A_{h} t}\right\|_{\mathscr{L}(H)} \leq \frac{c_{\theta} e^{\left(\omega_{0}+\varepsilon\right) t}}{t^{\theta}}, \quad t>0,0 \leq \theta \leq 1
$$

(the cases $0<\theta<1$ follow by interpolation from the endpoint cases $\theta=0$, $\theta=1$ ), with constant $c_{\theta}$ independent of $h$;

equivalent formulation in $\lambda$-domain: for $a>\omega_{0}$, there exists $\Sigma_{\text {app }}(A)=\Sigma_{\text {app }}\left(A ; a ; \theta_{a}\right)$, a closed triangular sector containing the axis $[-\infty, a]$ and delimited by the two rays $a+\rho e^{ \pm i \theta_{a}}$ for some $\pi / 2<\theta_{a} \leq \theta_{0}<2 \pi$,

associated with the analytic semigroup $e^{A t}$, and there exists $h_{a}$ such that, if $\Sigma^{c}$ denotes the complement of $\Sigma$ in $\mathbb{C}$, then for all $0<h \leq h_{a}$ we have

$$
\begin{gathered}
\sigma\left(A_{h}\right)=\text { spectrum of } A_{h} \subset \Sigma_{\text {app }}(A), \\
\left\|R\left(\lambda, A_{h}\right) A_{h}^{\theta}\right\|_{\mathscr{L}(H)} \leq \frac{C}{|\lambda-a|^{\theta}} \quad \forall \lambda \in \Sigma_{\text {app }}^{c}(A), \quad 0 \leq \theta \leq 1
\end{gathered}
$$

(the cases $0<\theta<1$ follow by interpolation from $\theta=0$ and $\theta=1$ ), $R(\lambda, \cdot)$ being the resolvent operator.

(A.2)

$$
\left\|\Pi_{h} \hat{A}^{-1}-\hat{A}_{h}^{-1} \Pi_{h}\right\|_{\mathscr{L}(H)} \leq C h^{s} \text { for some } s>0 \text { independent of } h .
$$

Approximation of $B$. We shall assume that the operators $B: U \rightarrow\left[\mathscr{D}\left(A^{*}\right)\right]^{\prime}$ and $B_{h}: U \rightarrow V_{h}$ satisfy the following approximating properties, where $\gamma$ and $s$ are defined by (1.3) and (1.15), respectively:

(A.3) ('inverse approximation property')

$$
\left\|B^{*}\left(\Pi_{h}-I\right) x\right\|_{U} \leq C h^{s(1-\gamma)}\|x\|_{\mathscr{D}\left(A^{*}\right)}, \quad x \in \mathscr{D}\left(A^{*}\right) .
$$

$$
\left\|B^{*} x_{h}\right\|_{U}+\left\|B_{h}^{*} x_{h}\right\|_{U} \leq C h^{-\gamma s}\left\|x_{h}\right\|_{H} \quad \forall x_{h} \in V_{h} .
$$

(If, in particular, we take $B_{h}=\Pi_{h} B$, then (A.5) is contained in (A.4).)

$$
\left\|B^{*} x-B_{h}^{*} \Pi_{h} x\right\|_{U} \leq C h^{s(1-\gamma)}\|x\|_{\mathscr{D}\left(A^{*}\right)}, \quad x \in \mathscr{D}\left(A^{*}\right) .
$$

Remark 1.1. Notice that assumptions (A.2)-(A.6) are standard approximation properties, where, moreover, in the case of spline approximations, $s$ is the order of the differential operator $A$. They are consistent with the regularity of the 
original operators $A$ and $B$. Moreover, they are satisfied by typical schemes (finite elements, finite differences, mixed methods, spectral approximations). The property of uniform analyticity (A.1) is not a standard assumption and needs to be verified in each case. However, to our knowledge, it is satisfied for most of the schemes and examples which arise from analytic semigroup problems. For instance, a sufficient condition for (A.1) to hold true is the uniform coercivity of the bilinear form associated with $A_{h}$ (see Lemma 4.2 in [14]). There are, however, a number of significant physical examples (e.g., structurally damped elastic systems), where the bilinear form is not coercive, while the underlying semigroups $e^{A_{h} t}$ are uniformly analytic (see $\S 6$ ).

1.2.2. Consequences of approximating assumptions on A. From (A.1) and (A.2), the following "rough" data estimates follow (see [14, Appendix] and [3]) (in a form to be used later):

(i) $\left\|e^{A_{h} t} \Pi_{h}-e^{A t}\right\|_{\mathscr{L}(H)}=\left\|e^{A_{h}^{*} t} \Pi_{h}-\Pi_{h} e^{A^{*} t}\right\|_{\mathscr{L}(H)} \leq C \frac{h^{s \theta} e^{\left(\omega_{0}+\varepsilon\right) t}}{t^{\theta}}$

$$
t>0,0 \leq \theta \leq 1, \forall \varepsilon>0 \text {; }
$$

(1.21) (ii) $\left\|R(\lambda, A)-R\left(\lambda, A_{h}\right) \Pi_{h}\right\|_{\mathscr{L}(H)} \leq C h^{s}, \quad s>0$,

uniformly in $\lambda \in \Sigma_{\text {app }}^{c}(A)$ (see definition of $\Sigma_{\text {app }}^{c}(A)$ below (1.14a));

(iii) $\left\|e^{A_{h}^{*} t} \Pi_{h}-\Pi_{h} e^{A^{*} t}\right\|_{\mathscr{L}\left(\mathscr{D}\left(A^{*}\right) ; H\right)} \leq C h^{s}$

uniformly in $t>0$ on compact intervals.

1.3. Approximation of dynamics and of control problem. Related Riccati equation. We now introduce an approximation of the control problem and of the corresponding Algebraic Riccati Equation.

Control problem. Given the approximating dynamics $y_{h}(t) \subset V_{h}$ satisfying

$$
\dot{y}_{h}(t)=A_{h} y_{h}(t)+B_{h} u(t), \quad y_{h}(0)=\Pi_{h} y_{0},
$$

minimize over all $u \in L_{2}(0, \infty ; U)$ the cost

$$
J\left(u, y_{h}\right)=\int_{0}^{\infty}\left[\left\|R y_{h}(t)\right\|_{Z}^{2}+\|u(t)\|_{U}^{2}\right] d t .
$$

It will be shown in $\S \S 4.1$ and 4.2 that the approximating dynamics (1.23) is stabilizable and detectable, in fact, uniformly in $h$. Thus, it is a standard finite-dimensional result (on $V_{h}$ ) -which is, in fact, contained in Theorem 1.0 when specialized to $V_{h}$-that there exists a unique Riccati approximating operator $P_{h}$, nonnegative self-adjoint, associated with (1.23), (1.24), solution of the following Algebraic Riccati Equation (A.R.E.) $)_{h}$ :

$$
\begin{gathered}
\left(A_{h}^{*} P_{h} x_{h}, y_{h}\right)_{H}+\left(P_{h} A_{h} x_{h}, y_{h}\right)_{H}+\left(R x_{h}, R y_{h}\right)_{Z} \\
=\left(B_{h}^{*} P_{h} x_{h}, B_{h}^{*} P_{h} y_{h}\right)_{U} \quad \forall x_{h}, y_{h} \in V_{h} .
\end{gathered}
$$


Equation (1.25) leads to a standard matrix Riccati equation for the numerical solution of which there exists a vast literature (see, e.g., [12]). Further properties of the approximating problem will be collected in $\$ 2.2$; see in particular the identity (2.20) for $P_{h}$.

\subsection{Main results of approximating schemes: Theorems $\mathbf{1 . 1}$ and $\mathbf{1 . 2}$.}

Theorem 1.1. Assume

I. the continuous hypotheses (1.3), $(S . C)=.(1.5),(D . C)=.(1.6)$, and , in addition,

(a) either $R>0$,

(b) or else $\hat{A}^{-1} K R: H \rightarrow H$ compact;

(a) either $B^{*} \widehat{A}^{*-1}: H \rightarrow U$ compact,

(b) or else $F: H \rightarrow U$ compact;

II. the approximation properties (1.3) and (A.1) $=(1.14)-(\mathrm{A} .6)=(1.19)$.

Then there exists $h_{0}>0$ such that for all $h<h_{0}$ the solution $P_{h}$ to $(A . R . E .)_{h}$ in (1.25) exists, is unique, and the following uniform bounds and convergence properties hold true:

(i)

$$
\left\|e^{A_{h, P_{h}}{ }^{t}}\right\|_{\mathscr{L}(H)} \leq C_{P} e^{-\bar{\omega}_{P} t}, \quad \bar{\omega}_{P}>0 \text { uniformly in } h
$$

(see Theorem 4.6, equation (4.27)), where

$$
A_{h, P_{h}}=A_{h}-B_{h} B_{h}^{*} P_{h} \text {. }
$$

(ii)

$$
\begin{aligned}
\left\|\left(\widehat{A}_{h}^{*}\right)^{1-\varepsilon} P_{h}\right\|_{\mathscr{L}(H)}+\left\|\left(\widehat{A}_{h}^{*}\right)^{1 / 2-\varepsilon} P_{h} \hat{A}_{h}^{1 / 2-\varepsilon}\right\|_{\mathscr{L}(H)} \leq C_{\varepsilon} \\
\forall \varepsilon>0, \text { uniformly in } h
\end{aligned}
$$

(see Theorem 4.7).

$$
\left\|P_{h} \Pi_{h}-P\right\|_{\mathscr{L}(H)} \leq C h^{\varepsilon_{0}} \rightarrow 0 \text { as } h \downarrow 0, \forall \varepsilon_{0}<s(1-\gamma)
$$

(see Theorem 5.1, equation (5.1)).

$$
\left\|B_{h}^{*} P_{h} \Pi_{h}-B^{*} P\right\|_{\mathscr{L}(H ; U)} \rightarrow 0 \text { as } h \downarrow 0
$$

(see Theorem 5.3, equation (5.5)).

(v) For all $\varepsilon_{0}<s(1-\gamma)$,

(1.33) $\sup _{t \geq 0} e^{\bar{\omega}_{P} t}\left\|u_{h}^{0}\left(t, \Pi_{h} x\right)-u^{0}(t, x)\right\|_{\mathscr{L}(H ; U)} \leq C h^{\varepsilon_{0}} \rightarrow 0 \quad$ as $h \downarrow 0, x \in H$ (see Corollary 5.5, equation (5.9)). 
(vi) For all $\varepsilon_{0}<s(1-\gamma)$,

$$
\left\|y_{h}^{0}\left(\cdot, \Pi_{h} x\right)-y^{0}(\cdot, \Pi x)\right\|_{\mathscr{L}\left(H ; L_{2}(0, \infty ; H)\right)} \leq C h^{\varepsilon_{0}} \rightarrow 0 \quad \text { as } h \downarrow 0
$$

(see Lemma 5.4, equation (5.7)).

(vii)

$$
\left\|y_{h}^{0}\left(\cdot, \Pi_{h} x\right)-y^{0}(\cdot, \Pi x)\right\|_{C([0, \infty] ; U)} \rightarrow 0 \quad \text { as } h \downarrow 0, x \in H
$$

(see Lemma 5.4, equation (5.8)).

(viii) For all $\varepsilon_{0}<s(1-\gamma)$ and for all $\varepsilon>0$,

$$
\sup _{t \geq 0} t^{\varepsilon} e^{\bar{\omega}_{P} t}\left\|y_{h}^{0}(t, x)-y^{0}(t, x)\right\|_{\mathscr{L}(H)} \leq C h^{\varepsilon_{0} \varepsilon} \rightarrow 0 \quad \text { as } h \downarrow 0
$$

(see Theorem 5.2, equation (5.4)).

(ix) For all $\varepsilon_{0}<s(1-\gamma)$,

$$
\left|J\left(u_{h}^{0}\left(\cdot, \Pi_{h} x\right), y_{h}^{0}\left(\cdot, \Pi_{h} x\right)\right)-J\left(u^{0}(\cdot, x), y^{0}(\cdot, x)\right)\right| \leq C h^{\varepsilon_{0}} \rightarrow 0
$$

(consequence of property (iii) in (1.31) and of (1.10) and (2.23)).

(x) Moreover, if in addition, for some $0<\theta<1, V_{h} \subset \mathscr{D}\left(\left(\widehat{A}^{*}\right)^{\theta}\right)$ and

$$
\left\|\left(\hat{A}^{*}\right)^{\theta} x_{h}\right\|_{H} \leq C_{\theta}\left\|\left(\hat{A}_{h}^{*}\right)^{\theta} x_{h}\right\|_{H} \text { or }\left(\hat{A}^{*}\right)^{\theta}\left(\hat{A}_{h}^{*-1}\right)^{\theta} \in \mathscr{L}\left(V_{h}, H\right),
$$

then (see Proposition 5.6, equations (5.11), (5.12)),

(1.39) $\left(\mathrm{x}_{1}\right)\left\|\left(\hat{A}^{*}\right)^{\theta}\left(P_{h} \Pi_{h}-P\right) x\right\|_{H} \rightarrow 0$ as $h \downarrow 0, x \in H, 0 \leq \theta<1$;

(1.40) $\quad\left(\mathrm{x}_{2}\right)\left\|\left(\hat{A}^{*}\right)^{\theta}\left(P_{h} \Pi_{h}-P\right) \hat{A}^{\theta} x\right\|_{H} \rightarrow 0$ as $h \downarrow 0, x \in H, 0 \leq \theta<\frac{1}{2}$.

Remark 1.2. Assumption (1.38) typically holds true with $\theta=\frac{1}{2}$. This is certainly the case when $A$ is coercive and $A_{h}$ is a standard Galerkin approximation of $A$, i.e., $\left(A_{h} x_{h}, y_{h}\right)_{H}=\left(A x_{h}, y_{h}\right)_{H}$.

Remark 1.3. If $A$ is self-adjoint (or, more generally, if $A=A_{1}+A_{2}$, with $A_{1}$ self-adjoint and $A_{2}: H \supset \mathscr{D}\left(\left(-A_{1}\right)^{1-\varepsilon}\right) \rightarrow H$ is bounded), one can take $\theta=\frac{1}{2}$ in (1.40).

Theorem 1.2. (i) The following uniform exponential stability holds true:

$$
\left\|e^{\left(A-B B_{h}^{*} P_{h}\right) t}\right\|_{\mathscr{L}(H)} \leq \widehat{C} e^{-\hat{\omega}_{P} t}, \quad \hat{\omega}_{P}>0,
$$

under the same assumptions as in Theorem 1.1.

(ii) Moreover,

$$
\sup _{t \geq 0} e^{\hat{\omega}_{P} t}\left\|e^{\left(A-B B_{h}^{*} P_{h}\right) t}-e^{\left(A-B B^{*} P\right) t}\right\|_{\mathscr{L}(H)} \rightarrow 0 \quad \text { as } h \downarrow 0 .
$$

Theorem 1.1 provides the basic convergence results (with rates) for the optimal solutions of the approximating problem (1.23), (1.24), the corresponding 
Riccati operators, and gain operators, to the same quantities of the original problem (1.1), (1.2).

The advantage of Theorem 1.2 is this: It states that the original system, once acted upon by the discrete feedback control law given by $u_{h}^{*}\left(t, \Pi_{h} x\right)=$ $-B^{*} P_{h} y_{h}^{*}\left(t, \Pi_{h} x\right)$, yields (uniformly) exponentially stable solutions (see also $[18, \S 4.3])$.

Remark 1.4. Instead of the original inner product $\left(x_{h}, y_{h}\right)_{H}$, one can introduce an equivalent inner product $\left(x_{h}, y_{h}\right)_{H_{h}}$, where $c_{1}\left\|x_{h}\right\|_{H} \leq\left\|x_{h}\right\|_{H_{h}} \leq c_{2}\left\|x_{h}\right\|_{H}$. In some situations, it is more convenient to work with a discrete inner product $(,)_{H_{h}}$ so as to simplify the computations for the adjoint operators for the discrete problem.

Remark 1.5. The literature on approximating schemes of optimal problems and related Riccati equations generally assumes (see [11])

(i) convergence properties of the "open loop" solutions, i.e., of the maps $u \rightarrow y$ of the continuous problem;

(ii) "uniform stabilizability/detectability" hypotheses for the approximating problems.

In contrast, our basic assumptions are:

(a) stabilizability/detectability hypotheses (S.C.)/(D.C.) of the continuous system;

(b) a "uniform analyticity" hypothesis (A.1) on the approximations.

Starting from (a) and (b), we then derive both the convergence properties of the open loop and the uniform stabilizability/detectability hypotheses-(i) and (ii) above-which are taken as assumptions in other treatments. Thus, the theory presented here is "optimal," in the sense that it assumes only what is strictly needed. Indeed, it can be shown that assumptions (A.1) and (S.C.)/(D.C.) are not only sufficient, but also necessary, for the main theorems presented here. These considerations are an important aspect of the entire theory, since, in the case where $B$ is an unbounded operator, the requirement, corresponding to (i) above in other treatments, of convergence $L_{h} \rightarrow L$ of the open loop solutions (see (2.1), (2.11) below) is a very strong assumption. Generally, even when $L$ is bounded and the scheme is consistent, it may well happen that the scheme is not even stable, i.e., $L_{h}$ may not be uniformly bounded in $h$. The properties of the composition $e^{A t} B$ may not be retained in the approximation $e^{A_{h} t} B_{h}$. Special care must be exercised in approximating $B$.

1.5. Literature. Within the literature concerned with approximation schemes for Algebraic Riccati Equations (A.R.E.) in infinite-dimensional spaces, we shall refer here only to works which focus on the case where the original free dynamics is modelled by an analytic semigroup $e^{A t}$, as in the present paper. Approximation results for parabolic problems with distributed controls, i.e., with the operator $B$ bounded $(\gamma=0$ in (1.3)), are given in [1]. Next, [18] analyzed 
the case of a parabolic problem defined on a bounded domain $\Omega \subset R^{n}$ with Dirichlet boundary control, via an abstract semigroup approach, where then $\gamma=\frac{3}{4}+\varepsilon$ in (1.3), i.e., the operator $\hat{A}^{-(3 / 4+\varepsilon)} B$ is bounded for all $\varepsilon>0$. This case may be viewed as a canonical illustration of the purely abstract situation where one has $\hat{A}^{-\gamma} B$ bounded for $\gamma<1$, and $A$ has compact resolvent. Thus, the treatment in [18] works equally well, mutatis mutandis, in the abstract case of an analytic semigroup generator $A$ with compact resolvent, and with $\hat{A}^{-\gamma} B$ bounded, $0 \leq \gamma<1$. There is a natural "cutting line" in the range of values of $\gamma$, which crucially bears on the degree of technical difficulties present in the treatment of the optimal control problem and its algebraic Riccati approximation: this is given by the special value $\gamma=\frac{1}{2}$.

Indeed, if $\hat{A}^{-\gamma} B$ is bounded with $\gamma<\frac{1}{2}$, then the corresponding input $\rightarrow$ solution operator $L$ is a priori continuous into $C([0, T] ; H)$, so that all the trajectories of the continuous dynamical system are a priori pointwise continuous in time, and the operator $B^{*} P$ is then a priori a bounded operator. Thus, in the case $\gamma<\frac{1}{2}$, a derivation of the A.R.E. may be given which closely parallels the pattern where $B$ is a bounded operator. (The same applies to the case $\gamma=\frac{1}{2}$ if $A$ is self-adjoint, or, more generally, it has a Riesz basis property on H.)

Instead, if $\hat{A}^{-\gamma} B$ is bounded, with $\frac{1}{2}<\gamma$, the operator $L$ is not continuous into $C([0, T] ; H)$, i.e., the open loop trajectories are generally not pointwise continuous in time. Here, a main technical difficulty is therefore to show that, nevertheless, the gain operator $B^{*} P$ is bounded. This is done by carefully analyzing the properties of the optimal solutions $y^{0}(t)$ (as distinguished from ordinary solutions $y(t)$ ) and by eventually showing via a boot strap argument that the optimal solutions $y^{0}(t)$ are pointwise continuous in time (unlike ordinary solutions $y(t)$ which are only, say, in $\left.L_{2}(0, T ; H)\right)$.

The strategy outlined above for the case $\gamma>\frac{1}{2}$-which was successfully implemented in [18] in the canonical case of a parabolic equation with Dirichlet boundary control, where $\gamma=\frac{3}{4}+\varepsilon$, and $A$ has compact resolvent-is also followed in the present abstract treatment, which moreover dispenses altogether with the assumption that $A$ has compact resolvent. This will cover, in a unified framework, some physically significant examples (see $\S 6$ ) of damped elastic systems, where, in fact, $A$ does not have compact resolvent. Thus, although much of the conceptual and technical developments of the present paper are a natural generalization of the arguments in [18], there are, however, also points of departure from [18] which require a different analysis, because of the now missing property that $A$ have compact resolvent (see Remark 5.1), which was naturally built in the parabolic problem [18]. Like [18], our treatment here uses, as a starting point, two sources: on the one hand, the properties of the continuous optimal control problem and related Algebraic Riccati Equation following the variational approach of [17]; and, on the other hand, the approximation results for analytic semigroups (see $[14,15,3])$. 
The importance of having a theory of approximation valid for $\gamma>\frac{1}{2}$ is fully justified by important physical problems, which are not solved by the direct, straightforward generalization from the case of $B$ bounded to the case of $\hat{A}^{-\gamma} B$ bounded with $\gamma \leq \frac{1}{2}$. Relevant examples where $\gamma>\frac{1}{2}$ include, in addition to parabolic problems with Dirichlet boundary control, also structurally damped elastic equations (see $\S 6$ ).

It was suggested from various sources that it would serve a useful purpose in the area to write a fully abstract explicit treatment of the general case $\gamma<1$ modelled after [18]. This is done in the present paper. Generally, we shall rely again on a combination of ideas and techniques of the continuous problem [17], together with general approximating properties of analytic semigroups $[14,15]$.

\section{BACKGROUND MATERIAL}

2.1. Continuous problem. In order to prove the main results, Theorems 1.1 and 1.2, we shall use explicit representation formulas (in operator form) which describe the optimal solution pair $\left\{u^{0}, y^{0}\right\}$ and the Riccati operator. The purpose of this section is to provide these representation formulas. To this end, we introduce the solution operator $L$ of problem (1.1) when $y_{0}=0$ :

$$
(L u)(t)=\int_{0}^{t} e^{A(t-\tau)} B u(\tau) d \tau:\left\{\begin{array}{c}
\text { continuous } L_{2}(0, T ; U) \\
\rightarrow L_{2}(0, T ; H) ; \\
\text { continuous } L_{\infty}(0, T ; U) \\
\rightarrow C\left([0, T] ; \mathscr{D}\left(\hat{A}^{1-\gamma}\right)\right) .
\end{array}\right.
$$

Its $L_{2}$-adjoint $L^{*}:(L u, v)_{L_{2}(0, T ; H)}=\left(u, L^{*} v\right)_{L_{2}(0, T ; U)}$ is given by

$$
\left(L^{*} v\right)(t)=B^{*} \int_{t}^{T} e^{A^{*}(\tau-t)} v(\tau) d \tau .
$$

We shall similarly introduce the corresponding operators related to the generator $-\widehat{A}=A-\omega I$, rather than to the generator $A$ :

$$
\begin{gathered}
(\widehat{L} u)(t)=\int_{0}^{t} e^{-\widehat{A}(t-\tau)} B u(\tau) d \tau: \text { continuous } L_{2}(0, \infty ; U) \\
\rightarrow L_{2}(0, \infty ; H) ; \\
\left(\widehat{L}^{*} v\right)(t)=B^{*} \int_{t}^{\infty} e^{-\widehat{A}^{*}(\tau-t)} v(\tau) d \tau: \text { continuous } L_{2}(0, \infty ; H) \\
\rightarrow L_{2}(0, \infty ; U) .
\end{gathered}
$$

With $\omega$ fixed once and for all, as in the Introduction below (1.2) in the standing assumption (ii), we introduce the notation

$$
\hat{u}^{0}\left(t, y_{0}\right)=e^{-\omega t} u^{0}\left(t, y_{0}\right), \quad \hat{y}^{0}\left(t, y_{0}\right)=e^{-\omega t} y^{0}\left(t, y_{0}\right),
$$

where $u^{0}\left(t, y_{0}\right), y^{0}\left(t, y_{0}\right)$ is the optimal pair of problem (1.1), (1.2), which originates at the point $y_{0}$ at time $t=0$. We set

$$
\widehat{\Phi}(t) x=\hat{y}^{0}(t, x)=e^{-\omega t} y^{0}(t, x)=e^{-\omega t} \Phi(t) x, \quad x \in H .
$$


Then, the optimal control and the corresponding optimal trajectory are given by the following explicit formulas [17, Theorem 2.4] (where the operator $\hat{A}$ in [17] corresponds to $-\widehat{A}$ in the present paper):

$$
\begin{aligned}
& \hat{y}^{0}\left(\cdot, y_{0}\right)=\widehat{\Phi}(\cdot) y_{0}=\left[I+\widehat{L} \widehat{L}^{*}\left(R^{*} R+2 \omega P\right)\right]^{-1}\left\{e^{-\widehat{A}^{*}} y_{0}\right\} \in L_{2}(0, \infty ; H) \\
& \begin{aligned}
-\hat{u}^{0}\left(\cdot, y_{0}\right) & =\left[I+\widehat{L}^{*}\left(R^{*} R+2 \omega P\right) \widehat{L}\right]^{-1} \widehat{L}^{*}\left[R^{*} R+2 \omega P\right]\left\{e^{-\widehat{A}^{*}} y_{0}\right\} \\
& \in L_{2}(0, \infty ; U) \\
& =\left\{\widehat{L}^{*}\left[R^{*} R+2 \omega P\right] \hat{y}^{0}(\cdot, x)\right\}(t)
\end{aligned}
\end{aligned}
$$

with inverses well defined in $L_{2}(0, \infty ; \cdot), \cdot=H$ or $U$. The solution $P$ to the A.R.E. in (1.8) satisfies the relation for $x \in H$ [17, Theorem 2.8] (where $\hat{A}$ in [17] corresponds to $-\widehat{A}$ now),

$$
P x=\int_{0}^{\infty} e^{-\widehat{A}^{*} t}\left[R^{*} R+2 \omega P\right] \widehat{\Phi}(t) x d t .
$$

2.2. Discrete problem. In order to describe the solution to the discrete problem (1.23), (1.24), we similarly introduce the operators,

$$
\begin{gathered}
\left(L_{h} u\right)(t)=\int_{0}^{t} e^{A_{h}(t-\tau)} B_{h} u(\tau) d \tau: \text { continuous } L_{2}(0, T ; U) \\
\rightarrow L_{2}\left(0, T ; V_{h}\right), \\
\left(L_{h}^{*} v\right)(t)=B_{h}^{*} \int_{t}^{T} e^{A_{h}^{*}(\tau-t)} \Pi_{h} v(\tau) d \tau,
\end{gathered}
$$

with $L_{h}^{*}$ being the $L_{2}$-adjoint of $L_{h}$ in the sense of (2.2), and finally,

$$
\begin{gathered}
\left(\widehat{L}_{h} u\right)(t)=\int_{0}^{t} e^{-\widehat{A}_{h}(t-\tau)} B_{h} u(\tau) d \tau: \text { continuous } L_{2}(0, \infty ; U) \\
\rightarrow L_{2}\left(0, \infty ; V_{h}\right), \\
\left(\widehat{L}_{h}^{*} v\right)(t)=B_{h}^{*} \int_{t}^{\infty} e^{-\widehat{A}_{h}^{*}(\tau-t)} \Pi_{h} v(\tau) d \tau: \text { continuous } L_{2}\left(0, \infty ; V_{h}\right) \\
\rightarrow L_{2}(0, \infty ; U),
\end{gathered}
$$

where we have defined, consistently with the continuous case below (1.2),

$$
\widehat{A}_{h}=-A_{h}+\omega I \text {. }
$$

Now let $\left\{u_{h}^{0}(t, x), y_{h}^{0}(t, x)\right\}$ be the optimal pair of the discrete optimal problem (1.23), (1.24), originating at the point $x \in V_{h}$ at the time $t=0$, and set

$$
\begin{gathered}
\hat{u}_{h}^{0}(t, x)=e^{-\omega t} u_{h}^{0}(t, x), \quad \hat{y}_{h}^{0}(t, x)=e^{-\omega t} y_{h}^{0}(t, x), \\
\widehat{\Phi}_{h}(t) x=\hat{y}_{h}^{0}(t, x)=e^{-\omega t} \Phi_{h}(t) x, \quad x \in V_{h},
\end{gathered}
$$


consistently with (2.6), (2.7). Then, the discrete optimal pair of problem (1.23), (1.24), is given by the following explicit formulas with $y_{0 h}=\Pi_{h} y_{0} \in V_{h}$, which are the counterpart of formulas (2.8), (2.9) in the continuous case [18, p. 192]:

$$
\begin{aligned}
\hat{y}_{h}^{0}\left(\cdot, y_{0 h}\right) & =\widehat{\Phi}_{h}(\cdot) y_{0 h}=\left[I+\widehat{L}_{h} \widehat{L}_{h}^{*} \Pi_{h}\left(R^{*} R+2 \omega P_{h}\right)\right]^{-1}\left\{e^{-\widehat{A}_{h} \cdot} y_{0 h}\right\} \\
& \in L_{2}\left(0, \infty ; V_{h}\right)
\end{aligned}
$$

$$
\begin{aligned}
-\hat{u}_{h}^{0}\left(\cdot, y_{0 h}\right) & =\left[I+\widehat{L}_{h}^{*} \Pi_{h}\left(R^{*} R+2 \omega P_{h}\right) \widehat{L}_{h}\right]^{-1} \widehat{L}_{h}^{*} \Pi_{h}\left[R^{*} R+2 \omega P_{h}\right]\left\{e^{-\widehat{A}_{h}^{*}} y_{0 h}\right\} \\
& \in L_{2}(0, \infty ; U) .
\end{aligned}
$$

The corresponding Riccati operator $P_{h}$ satisfies

$$
P_{h} x=\int_{0}^{\infty} e^{-\widehat{A}_{h}^{*} t} \mathrm{iI}_{h}\left[R^{*} R+2 \omega P_{h}\right] \widehat{\Phi}_{h}(t) x d t, \quad x \in V_{h} .
$$

The proofs of our main convergence results are based on a careful analysis of the convergence properties of the basic operators $\widehat{L}_{h}$ and $\widehat{L}_{h}^{*}$ to be given in §3. A few more formulas to be invoked later are:

$$
\begin{gathered}
\hat{y}_{h}^{0}(t, x)=e^{-\widehat{A}_{h} t} x+\left\{\widehat{L}_{h} \hat{u}_{h}^{0}(\cdot, x)\right\}(t), \quad x \in V_{h}, \\
-\hat{u}_{h}^{0}(t, x)=\left\{\widehat{L}_{h}^{*}\left[R^{*} R+2 \omega P_{h}\right] \hat{y}_{h}^{0}(\cdot, x)\right\}(t), \quad x \in V_{h},
\end{gathered}
$$

counterparts of (2.8) and (2.9b), and finally, the counterpart of (1.10),

$$
J\left(u_{h}^{0}(\cdot, x), y_{h}^{0}(\cdot, x)\right)=\left(P_{h} x, x\right), \quad x \in V_{h} .
$$

\section{CONVERGENCE PROPERTIES OF THE OPERATORS}

$$
L_{h} \text { AND } L_{h}^{*} ; \widehat{L}_{h} \text { AND } \widehat{L}_{h}^{*}
$$

Lemma 3.1. Let assumptions (A.1) $=(1.14)$ through (A.6) $=(1.19)$ hold true. Then we have for all $0<h \leq h_{0}$, with constants independent of $h$ :

(i) $\left\|B_{h}^{*} e^{A_{h}^{*} t} \Pi_{h}-B^{*} e^{A^{*} t}\right\|_{\mathscr{L}(H ; U)} \leq C \frac{h^{s(1-\gamma)}}{t} e^{\left(\omega_{0}+\varepsilon\right) t}, \quad t>0 ;$

(ii) $\left\|B_{h}^{*} e^{A_{h}^{*} t} \Pi_{h}-B^{*} e^{A^{*} t}\right\|_{\mathscr{L}\left(\mathscr{D}\left(\widehat{A}^{*}\right) ; U\right)} \leq C h^{s(1-\gamma)} e^{\left(\omega_{0}+\varepsilon\right) t}$;

and by interpolation between (3.1) and (3.2), with $0 \leq \theta \leq 1$,

(iii) $\left\|B_{h}^{*} e^{A_{h}^{*} t} \Pi_{h}-B^{*} e^{A^{*} t}\right\|_{\mathscr{L}\left(\mathscr{D}\left(\left(\widehat{A}^{*}\right)^{\theta}\right) ; U\right)} \leq C_{\theta} \frac{h^{s(1-\gamma)}}{t^{1-\theta}} e^{\left(\omega_{0}+\varepsilon\right) t}, \quad t>0$.

Moreover,

(iv) $\left\|B_{h}^{*} e^{A_{h}^{*} t} \Pi_{h}\right\|_{\mathscr{L}(H ; U)} \leq C \frac{e^{\left(\omega_{0}+\varepsilon\right) t}}{t^{\gamma}}, \quad t>0$;

$$
\begin{aligned}
\left\|B_{h}^{*} e^{A_{h}^{*} t} \Pi_{h}-B^{*} e^{A^{*} t}\right\|_{\mathscr{L}(H ; U)} \leq & C \frac{h^{s(1-\gamma) \theta}}{t^{\theta+(1-\theta) \gamma}} e^{\left(\omega_{0}+\varepsilon\right) t}, \\
t & >0,0 \leq \theta \leq 1 ;
\end{aligned}
$$


and by interpolation between (3.5) and (3.2), with $0 \leq r \leq 1$,

$$
\begin{aligned}
& \text { (vi) }\left\|B_{h}^{*} e^{A_{h}^{*} t} \Pi_{h}-B^{*} e^{A^{*} t}\right\|_{\mathscr{L}\left(\mathscr{D}\left(\left(\widehat{A}^{*}\right)^{r}\right) ; U\right)} \leq \frac{C h^{s(1-\gamma)[r+(1-r) \theta]}}{t^{(1-r)[\theta+\gamma(1-\theta)]}} e^{\left(\omega_{0}+\varepsilon\right) t} \text {, } \\
& t>0,0 \leq \theta \leq 1 \text {. }
\end{aligned}
$$

Proof. In the Supplement section.

The proofs of the next two main results of this section are given in the Supplement.

Theorem 3.2. With reference to the operators defined in (2.1), (2.3), (2.11), (2.12), we have the following results under the assumptions of Lemma 3.1, where $0<\theta<1$ is arbitrary and $0<h \leq h_{0}$ :

$$
\text { (i) }\left\|L_{h}-L\right\|_{L_{2}}=\left\|L_{h}^{*}-L^{*}\right\|_{L_{2}} \leq C_{T} h^{s(1-\gamma) \theta} \text {, }
$$

where the first norm is in $\mathscr{L}\left(L_{2}(0, T ; U) ; L_{2}(0, T ; H)\right)$ and similarly for the second norm with $U$ and $H$ interchanged;

$$
\text { (ii) }\left\|L_{h}-L\right\|_{\mathscr{L}\left(L_{\infty}(0, T ; U) ; C([0, T] ; H)\right)} \leq C_{T} h^{s(1-\gamma) \theta} .
$$

Theorem 3.3. With reference to the operators defined in (2.4), (2.5) and (2.13), (2.14), the following convergence results hold true under the assumptions of Lemma 3.1 , where $0 \leq \theta<1$ is arbitrary:

$$
\text { (i) }\left\|\widehat{L}_{h}-\widehat{L}\right\|_{L_{2}}=\left\|\widehat{L}_{h}^{*}-\widehat{L}^{*}\right\|_{L_{2}} \leq C_{\theta} h^{s(1-\gamma) \theta} \rightarrow 0 \text { as } h \downarrow 0 \text {, }
$$

where the first norm is in $\mathscr{L}\left(L_{2}(0, \infty ; U) ; L_{2}(0, \infty ; H)\right)$, while the second norm is similar with $U$ and $H$ interchanged;

$$
\text { (ii) }\left\|\widehat{L}_{h}-\widehat{L}\right\|_{\infty, C}=\left\|\widehat{L}_{h}^{*}-\widehat{L}^{*}\right\|_{\infty, C} \leq C h^{s(1-\gamma) \theta} \rightarrow 0 \text { as } h \downarrow 0 \text {, }
$$

where the first norm is in $\mathscr{L}\left(L_{\infty}(0, \infty ; U) ; C([0, \infty] ; H)\right)$, while the second norm is similar with $U$ and $H$ interchanged.

\section{Perturbation Results}

The goal of the first two subsections is to show that the properties of analyticity and exponential stability of the semigroup $e^{A_{F} t}$ are preserved, uniformly in $h$, by its approximations.

4.1. Uniform analyticity and uniform exponential stability of the operators $A_{h, F_{h}}$ $=A_{h}+B_{h} F_{h}$ and $A_{F_{h}}=A+B F_{h}$.

4.1.1. Uniform analyticity. Throughout this subsection, we let $F \in \mathscr{L}(H ; U)$, and we consider the operator

$$
A_{F} \equiv A+B F: H \supset \mathscr{D}\left(A_{F}\right) \rightarrow H
$$

which, in view of the standing assumption (1.3), generates likewise an s.c., analytic semigroup $e^{A_{F} t}$ on $H, t \geq 0$ (as justified below (1.5)). In later sections, 
but not in this subsection, we shall consider the case where $F$ is a stabilizing feedback operator. We let

$$
\begin{aligned}
\Sigma\left(A_{F}\right)= & \Sigma\left(A_{F} ; a_{F} ; \theta_{F}\right) \\
= & (\text { closed }) \text { triangular sector containing the axis } \\
& {\left[-\infty, a_{F}\right] \text { and delimited by the two rays } a_{F}+\rho e^{ \pm i \theta_{F},} } \\
& 0 \leq \rho<\infty, \text { for some } \theta_{F}, \pi / 2<\theta_{F}<2 \pi,
\end{aligned}
$$

such that the spectrum $\sigma\left(A_{F}\right) \subset \Sigma\left(A_{F}\right)$. For a stabilizing $F$ as in the assumption (S.C.) $=(1.5)$, we have $a_{F}=-\omega_{F}<0$ in the notation of (1.5). In comparing $\Sigma\left(A_{F}\right)$ with the sector $\Sigma_{\text {app }}(A) \supset \sigma(A)$ in $\S 1.2 .2$, we may say that we can always assume without loss of generality that one sector is contained in the other: If $a_{F} \leq a$, then we can choose $\theta_{a}<\theta_{F}$, and then $\Sigma(F) \subset \Sigma_{\text {app }}(A)$; instead, if $a_{F}>a$, we can choose $\theta_{a}>\theta_{F}$, and then $\Sigma(F) \supset \Sigma_{\text {app }}(A)$. The first instance with $a_{F}=-\omega_{F}<a$ occurs if $F$ is a stabilizing feedback operator. For the sake of definiteness, in the lemma below we shall assume that $a_{F} \leq a$, and so $\Sigma(F) \subset \Sigma_{\text {app }}(A)$, the case which arises with $F$ a stabilizing operator. Next, we consider the approximation of $A_{F}$ defined by

$$
A_{h, F_{h}} \equiv A_{h}+B_{h} F_{h}: V_{h} \rightarrow V_{h}
$$

with $F_{h} \in \mathscr{L}\left(V_{h} ; U\right)$ and $A_{h}, B_{h}$ as in $\S 1.2 .1$.

The next result shows that if $\left\{\left\|F_{h}\right\|\right\}$ is uniformly bounded in $h$, then the operators $A_{h, F_{h}}$ defined by (4.3) generate "uniformly" analytic semigroups on $H$. With the stipulation above (4.3), we have the following lemma, whose proof is in the Supplement section.

Lemma 4.1. Let $\left\|F_{h}\right\|_{\mathscr{L}\left(V_{h} ; U\right)} \leq$ const, uniformly in $h$. Then, given $1>\delta>0$, there exist $r_{\delta}>0$ and $h_{\delta}>0$ such that for a suitable $\Sigma_{\text {app }}^{c}(A)=\Sigma_{\text {app }}^{c}\left(A ; a, \theta_{a}\right)$

$$
\text { (i) } \begin{aligned}
\left\|R\left(\lambda, A_{h, F_{h}}\right)\right\|_{\mathscr{L}\left(V_{h}\right)} \leq & \frac{1}{1-\delta}\left\|R\left(\lambda, A_{h}\right)\right\|_{\mathscr{L}\left(V_{h}\right)} \\
& \forall \lambda \in \Sigma_{\text {app }}^{c}(A),|\lambda| \geq r_{\delta}, \\
& \forall h, 0<h<h_{\delta} \leq h_{a} ; \\
\text { (ii) }\left\|R\left(\lambda, A_{h, F_{h}}\right)\right\|_{\mathscr{L}\left(V_{h}\right)} \leq & \frac{C}{1-\delta} \frac{1}{|\lambda-a|},
\end{aligned}
$$

$\lambda$ and $h$ as in (4.4);

$$
\text { (iii) }\left\|R\left(\lambda, A_{h, F_{h}}\right) A_{h, F_{h}}^{\theta}\right\|_{\mathscr{L}\left(V_{h}\right)} \leq \frac{C}{1-\delta} \frac{1}{|\lambda-a|^{1-\theta}}, \quad 0 \leq \theta \leq 1,
$$

$\lambda$ and $h$ as in (4.4).

Remark 4.1. Lemma 4.1 on uniform analyticity holds true also for the operators

$$
A_{F_{h}} \equiv A+B F_{h}, \quad \mathscr{D}\left(A_{F_{h}}^{*}\right)=\mathscr{D}\left(\left(\hat{A}^{*}\right)^{\gamma}\right),
$$

in which case the proof is simpler. 
4.1.2. Uniform exponential stability of $A_{h, F_{h}}$ and $A_{F_{h}}$. In this subsection we assume explicitly the stabilizability condition (S.C.) $=(1.5)$ that $F \in \mathscr{L}(H ; U)$ is stabilizing, i.e.,

$$
\left\|e^{A_{F} t}\right\|_{\mathscr{L}(H)} \subseteq M_{F} e^{-\omega_{F} t}, \quad t \geq 0, \omega_{F}>0 .
$$

We shall prove in the Supplement section that $e^{A_{h, F_{h}}{ }^{t}}$ and $e^{A_{F_{h}}{ }^{t}}$ are uniformly exponentially stable.

Theorem 4.2. Assume (4.8) and, moreover, that with $\left\|F_{h}\right\| \leq$ const, we have

$$
\left\|R\left(\lambda_{0}, A\right) B\left(F_{h} \Pi_{h}-F\right)\right\|_{\mathscr{L}(H)} \rightarrow 0 \text { as } h \downarrow 0 \text {, for some } \lambda_{0} \in \rho(A) \text {. }
$$

Then, given $\varepsilon>0$, there exists $h_{\varepsilon}>0$ and a three-sided sector $\Sigma_{\text {app }}\left(A_{F}\right)$, which may be taken to be

$$
\Sigma_{\text {app }}\left(A_{F}\right)=\Sigma_{\text {app }}(A) \cap\left\{\operatorname{Re} \lambda \leq-\omega_{F}+\varepsilon\right\},
$$

$\Sigma_{\text {app }}(A)=\Sigma_{\text {app }}\left(A ; a ; \theta_{a}\right)$ for some $a>\omega_{0}$, such that for all $0<h \leq h_{\varepsilon} \leq h_{a}$, the operators in (4.3) satisfy

(i) $\sigma\left(A_{h, F_{h}}\right) \subset \Sigma_{\text {app }}\left(A_{F}\right)$;

(ii) $\left\|e^{A_{h, F_{h}} \|}\right\|_{\mathscr{L}\left(V_{h}\right)} \leq M_{1 \varepsilon} e^{\left(-\omega_{F}+\varepsilon\right) t}, \quad t \geq 0$;

$$
\begin{aligned}
\left\|R\left(\lambda, A_{h, F_{h}}\right) A_{h, F_{h}}^{\beta}\right\|_{\mathscr{L}\left(V_{h}\right)} \leq & \frac{C}{\left|\lambda+\omega_{F}-\varepsilon\right|^{1-\beta}}, \\
& \lambda \in \Sigma_{\text {app }}^{c}\left(A_{F}\right), 0 \leq \beta \leq 1,
\end{aligned}
$$

uniformly in $h$. Thus, $e^{A_{h, F_{h}}{ }^{t}}$ is uniformly exponentially stable and, a fortiori from Lemma 4.1, uniformly analytic.

Remark 4.2. We note explicitly that either one of the following conditions is sufficient for assumption (4.9) to hold true:

(i) either $F_{h}^{*} \rightarrow F^{*}$ strongly, and $B^{*} R\left(\lambda_{0}, A^{*}\right)$ compact $H \rightarrow U$ (as assumed in (1.27a)) (see [13, p. 151]);

(ii) or else $F_{h} \Pi_{h} \rightarrow F$ in the uniform operator norm $\mathscr{L}(H ; U)$.

Remark 4.3. Theorem 4.2 holds true also for the operators $A_{F_{h}}=A+B F_{h}$ in (4.7), where, in fact, a simpler proof applies.

Remark 4.4. The role between assumption (4.8) and conclusion (4.12) can be reversed. The same proof yields the following result. Let

$$
\left\|e^{A_{h . F_{h}}{ }^{t}}\right\|_{\mathscr{L}(H)} \leq M e^{-\delta t}, \quad \delta>0
$$

(instead of (4.8)), and assume the convergence property (4.9) as before. Then, we obtain the conclusions corresponding to (4.11), (4.12), (4.13), with $A_{h, F_{h}}$ replaced by $A_{F}$; in particular, we obtain

$$
\left\|e^{A_{F} t}\right\| \leq \bar{M} e^{-\bar{\omega}_{F} t}, \quad \bar{\omega}_{F}>0 .
$$


4.2. Uniform detectability of the generators $A_{h, K}=A_{h}+\Pi_{h} K R \Pi_{h} ; L_{2}$-stability of $L_{h, K} B_{h}$ and $L_{h, K} \Pi_{h} K$. Throughout this subsection, we consider the operator

$$
A_{K}=A+K R, \quad H \supset \mathscr{D}\left(A_{K}\right) \rightarrow H,
$$

with $K \in \mathscr{L}(Z ; H)$ satisfying the detectability condition (D.C.) $=(1.6)$, so that

$$
\left\|e^{A_{K} t}\right\|_{\mathscr{L}(H)} \leq M_{K} e^{-\omega_{K} t} \text { for some } \omega_{K}>0 .
$$

Moreover, we assume throughout that $R\left(\lambda_{0}, A\right) K R$ : compact $H \rightarrow H$, which is hypothesis $(1.26 \mathrm{~b})$ of Theorem 1.1. We take the following approximations,

$$
A_{h, K}=A_{h}+\Pi_{h} K R \Pi_{h}: V_{h} \rightarrow V_{h},
$$

i.e., $R_{h}=R \Pi_{h} \rightarrow R$ strongly, $K_{h}=\Pi_{h} K \rightarrow K$ strongly. Then in view of the compactness assumption above, we have $R\left(\lambda_{0}, A\right) K R\left(\Pi_{h}-I\right) \rightarrow 0$ in the uniform norm $\mathscr{L}(H)$, and from Theorem 4.2 and Lemma 4.1, we obtain at once

Proposition 4.3 (Uniform detectability). The semigroups $e^{A_{h, K^{t}}}$ are uniformly analytic and, moreover, uniformly exponentially stable in $h$. Given $\varepsilon>0$, there exist $h_{\varepsilon}>0$ and a three-sided sector

$$
\Sigma_{\text {app }}\left(A_{K}\right) \equiv \Sigma_{\text {app }}(A) \cap\left\{\operatorname{Re} \lambda \leq-\omega_{K}+\varepsilon\right\}
$$

such that

$$
\sigma\left(A_{k, K}\right) \subset \Sigma_{\text {app }}\left(A_{k}\right)
$$

and

$$
\left\|e^{A_{h, K^{t}}}\right\|_{\mathscr{L}(H)} \leq M_{K, \varepsilon} e^{\left(-\omega_{K}+\varepsilon\right) t}, \quad t>0 .
$$

The following perturbation result will be invoked later.

Lemma 4.4. For any $1>\delta>0$, there is $r_{\delta}>0$ such that

$$
\left\|B_{h}^{*} R\left(\lambda, A_{h, K}^{*}\right)\right\|_{\mathscr{L}(H ; U)} \leq \frac{\text { const }}{(1-\delta) r_{\delta}^{1-\gamma}}, \quad \lambda \in \Sigma_{\text {app }}^{c}\left(A_{K}\right),|\lambda| \geq r_{\delta} .
$$

Proof. Since, as we say in (S.4.4) of the Supplement,

$$
R\left(\lambda, A_{h, K}\right)=\left[I-R\left(\lambda, A_{h}\right) \Pi_{h} K R \Pi_{h}\right]^{-1} R\left(\lambda, A_{h}\right),
$$

an estimate like (S.4.5) holds true for the inverse term [ $]^{-1}$ for $|\lambda| \geq r_{\delta}$, and thus, taking adjoints,

$$
\begin{aligned}
\left\|B_{h}^{*} R\left(\lambda, A_{h, K}^{*}\right)\right\|_{\mathscr{L}(H ; U)} & \leq \frac{1}{1-\delta}\left\|B_{h}^{*} R\left(\lambda, A_{h}^{*}\right)\right\|_{\mathscr{L}(H ; U)} \\
& \leq \frac{1}{1-\delta} \frac{C}{|\lambda-a|^{1-\gamma}}, \quad \lambda \text { as in (4.20), }
\end{aligned}
$$

recalling (S.4.2) of the Supplement, and (4.20) follows from (4.21). 
As a corollary to Lemma 4.4, we shall derive a stability result involving the approximating operators

$$
\left(L_{h, K} f_{h}\right)(t) \equiv \int_{0}^{t} e^{A_{h, K}(t-\tau)} f_{h}(\tau) d \tau: L_{2}\left(0, \infty ; V_{h}\right) \rightarrow \text { itself. }
$$

Corollary 4.5. With reference to (4.22), we have

(i) $\left\|L_{h, K} B_{h} u\right\|_{L_{2}(0, \infty ; H)} \leq C\|u\|_{L_{2}(0, \infty ; U)}$,

$$
\text { (ii) }\left\|L_{h, K} \Pi_{h} K z\right\|_{L_{2}(0, \infty ; H)} \leq C\|z\|_{L_{2}(0, \infty ; Z)} \text {. }
$$

Proof. (i) By the Parseval equality, it suffices to show that

$$
\left\|R\left(\lambda, A_{h, K}\right) B_{h} \hat{u}(\lambda)\right\|_{H} \leq C\|\hat{u}(\lambda)\|_{U}
$$

for all $\{\lambda: \operatorname{Re} \lambda=0\} \subset \Sigma_{\text {app }}^{c}\left(A_{K}\right)$. But (4.25) holds true by duality on (4.20). The proof for (ii) is identical.

4.3. Uniform stability of the feedback semigroup $\exp \left(A_{h, P_{h}} t\right)$. Let $P$ be the Riccati operator asserted by Theorem 1.0. With reference to the approximating optimal control problem (1.23), (1.24), we may now say-on the basis of the results of $\S \S 4.1$ and 4.2 -that the approximating dynamics $(1.23)$ is stabilizable and detectable, in fact uniformly in $h$. Thus, it is a standard result (contained in Theorem 1.0 when interpreted on $V_{h}$ ) that there exists a unique Riccati (approximating) operator $P_{h}$ associated with (1.23), (1.24), solution of the (A.R.E.) $)_{h}$ in (1.25). The goal of this subsection is to prove (in the Supplement) that the corresponding operator

$$
A_{h, P_{h}} \equiv A_{h}-B_{h} B_{h}^{*} P_{h}
$$

satisfies the uniform exponential stability condition (1.28) of Theorem 1.1.

Theorem 4.6 (Uniform stability of $e^{A_{h, P_{h}}{ }^{t}}$ ). Under the sets of assumptions I and II of Theorem 1.1, there exist $\bar{\omega}_{P}>0, \bar{M}_{P}>0$ such that

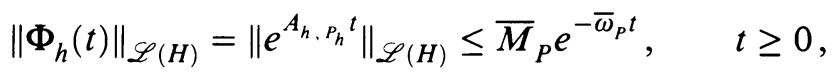

thereby proving (1.28) of Theorem 1.1. Recall from (4.26) and (2.16) that $\exp \left(A_{h, P_{h}}\right) t=\Phi_{h}(t)$.

4.4. Uniform regularity of $P_{h}$. We recall that $P_{h}$ is given, as described at the beginning of $\S 4.3$. In this section we shall show (in the Supplement) that $P_{h}$ is uniformly bounded not only in $\mathscr{L}(H)$, as already claimed by Lemma S.4.3 of the Supplement, equation (S.4.19), but in fact in a stronger norm. In particular, we shall prove statement (1.30) of the main Theorem 1.1.

Theorem 4.7. We have, uniformly in $h \downarrow 0$,

$$
\text { (i) }\left\|\left(\hat{A}_{h}^{*}\right)^{\theta} P_{h}\right\|_{\mathscr{L}(H)} \leq \text { const }_{\theta}, \quad 0 \leq \theta<1 \text {; }
$$$$
\text { (ii) }\left\|B_{h}^{*} P_{h}\right\|_{\mathscr{L}(H ; U)} \leq \text { const; }
$$$$
\text { (iii) }\left\|\left(\widehat{A}_{h}^{*}\right)^{\theta} P_{h} \widehat{A}_{h}^{\theta}\right\|_{\mathscr{L}(H)} \leq \text { const }_{\theta}, \quad 0 \leq \theta<\frac{1}{2} \text {; }
$$ 
or equivalently,

$$
\left\|P_{h}\right\|_{\mathscr{L}\left(\mathscr{D}\left(\left(\widehat{A}_{h}^{*}\right)^{*}\right) ;\left[\mathscr{D}\left(\left(\hat{A}_{h}^{*}\right)^{\theta}\right)\right]^{\prime}\right)} \leq \text { const }_{\theta} .
$$

Corollary 4.8. If for some $\theta<1$ we have

$$
\left\|\left(\hat{A}^{*}\right)^{\theta} x_{h}\right\|_{h} \leq C\left\|\left(\hat{A}_{h}^{*}\right)^{\theta} x_{h}\right\|_{H} \quad \forall x_{h} \in V_{h},
$$

then

$$
\left\|\left(\widehat{A}^{*}\right)^{\theta} P_{h}\right\|_{\mathscr{L}(H)} \leq \text { const }_{\theta}
$$

and, consequently,

$$
\left\|\left(\widehat{A}^{*}\right)^{\theta} P_{h} \hat{A}^{\theta}\right\|_{\mathscr{L}(H)} \leq \text { const }_{\theta}, \quad 0 \leq \theta<\frac{1}{2} .
$$

Remark 4.5. Assumption (4.31) holds true with any $\theta \leq \frac{1}{2}$ for Galerkin approximations.

The operators $A_{h, P_{h}}$ generate a family of semigroups $e^{A_{h} P_{h}{ }^{t}}$ which are uniformly stable by Theorem 4.6. They are also uniformly analytic by the next corollary.

Corollary 4.9. The operators $A_{h, P_{h}}=A_{h}-B_{h} B_{h}^{*} P_{h}$ in (4.26) generate a uniformly analytic family $e^{A_{h, P_{h}{ }^{t}}}$ of semigroups.

Proof. We just apply Lemma 4.1 with $F_{h}=B_{h}^{*} P_{h}$, which is legal by the uniform estimate (4.29) of Theorem 4.7.

\section{UNIFORM CONVERGENCE $P_{h} \Pi_{h} \rightarrow P$}

\subsection{Uniform convergence $P_{h} \Pi_{h} \rightarrow P$ of Riccati operators.}

Theorem 5.1 (Property (1.31) of main Theorem 1.1). For any $\varepsilon_{0}<s(1-\gamma)$,

$$
\left\|P_{h} \Pi_{h}-P\right\|_{\mathscr{L}(H)} \leq C h^{\varepsilon_{0}} \rightarrow 0 \text { as } h \downarrow 0 .
$$

The proof of Theorem 5.1 is given in the Supplement. It is based, among other things, on the following four operators:

$$
\begin{array}{cl}
A_{P}=A-B B^{*} P, & A_{h, P}=A_{h}-B_{h} B^{*} P, \\
A_{P_{h}}=A-B B_{h}^{*} P_{h}, & A_{h, P_{h}}=A_{h}-B_{h} B_{h}^{*} P_{h} .
\end{array}
$$

The first and the fourth, which were already defined by (1.12) and (4.26), refer to optimal dynamics, continuous and discrete. The second and third are defined here for the first time. They define competitive dynamics. 
As a corollary of part of the proof of Theorem 5.1, we obtain (see Supplement)

Theorem 5.2 (Property (1.36) of main Theorem 1.1). For any $\varepsilon>0$ we have

$$
\begin{gathered}
\sup _{0 \leq t} e^{\bar{\omega}_{P} t} t^{\varepsilon}\left\|e^{A_{h, P_{h}} t} \Pi_{h}-e^{A_{P} t}\right\|_{\mathscr{L}(H)} \\
\leq C h^{\varepsilon_{0} \varepsilon} \rightarrow 0 \quad \text { as } h \downarrow 0, \forall \varepsilon_{0}<s(1-\gamma), \\
\Phi(t) x=e^{A_{P} t} x=y^{0}(\cdot, x), \quad \Phi_{h}(t) \Pi_{h} x=e^{A_{h, P_{h}}{ }^{t} \Pi_{h} x=y_{h}^{0}\left(\cdot, \Pi_{h} x\right) .}
\end{gathered}
$$

Remark 5.1. The present proof of Theorem 5.1 is somewhat different from that in [18] and, moreover, it applies to the case of more general approximating assumptions (not necessarily Galerkin) without requiring that $A$ has compact resolvent as in [18]. One may also extend to the present case of $B$ unbounded the original approach of [11] given there for $B$ bounded, but this route-based on the finite time problem-is much longer.

\subsection{Uniform convergence $B_{h}^{*} P_{h} \Pi_{h} \rightarrow B^{*} P$ of gain operators.}

Theorem 5.3 (Property (1.32) of main Theorem 1.1). We have

$$
\left\|B_{h}^{*} P_{h} \Pi_{h}-B^{*} P\right\|_{\mathscr{L}(H ; U)} \rightarrow 0 \text { as } h \downarrow 0,
$$

where the rate is $h^{\varepsilon_{0}}, \varepsilon_{0}<s(1-\gamma)$ if $\gamma<\frac{1}{2}$.

The proof of Theorem 5.3, given in the Supplement, requires the following lemma.

Lemma 5.4 (Properties (1.33), (1.36), (1.37) of main Theorem 1.1). We have for $\varepsilon_{0}<s(1-\gamma)$ :

$$
\left\|u_{h}^{0}\left(\cdot, \Pi_{h} x\right)-u^{0}(\cdot, x)\right\|_{\mathscr{L}\left(H ; L_{2}(0, \infty ; U)\right)} \leq C h^{\varepsilon_{0}} \rightarrow 0 \quad \text { as } h \downarrow 0,
$$

equivalently by (S.4.20) with reference to (5.3),

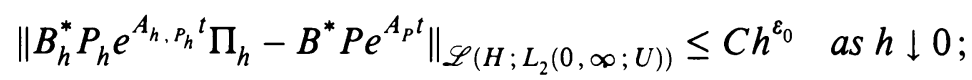

$$
\left\|\Phi_{h}(\cdot) \Pi_{h}-\Phi(\cdot)\right\|_{\mathscr{L}\left(H ; L_{2}(0, \infty ; H)\right)} \leq C h^{\varepsilon_{0}} \quad \text { as } h \downarrow 0 ;
$$

(iii) for any finite $0<T<\infty$, the following result, which complements (5.5), holds true:

$$
\left\|\left[\Phi_{h}(\cdot) \Pi_{h}-\Phi(\cdot)\right] x\right\|_{C([0, \infty] ; H)} \rightarrow 0 \text { as } h \downarrow 0, x \in H,
$$

where $y_{h}^{0}\left(\cdot, \Pi_{h} x\right)=\Phi_{h}(\cdot) \Pi_{h} x, y^{0}(\cdot, x)=\Phi(\cdot) x \quad($ see $(2.17),(2.7))$.

5.3. Uniform convergence $u_{h}^{0} \rightarrow u^{0}$.

Corollary 5.5 (Property (1.33) of main Theorem 1.1). We have (see Supplement) (5.9) $\left\|u_{h}^{0}\left(\cdot, \Pi_{h} x\right)-u^{0}(\cdot, x)\right\|_{\mathscr{L}(H ; C((0, \infty) ; U))} \leq C h^{\varepsilon_{0}} e^{-\bar{\omega}_{P} t}$ as $h \downarrow 0, x \in H$. 
5.4. Convergence $\left(\widehat{A}^{*}\right)^{\theta}\left(P_{h} \Pi_{h}-P\right) x \rightarrow 0$. So far, we have shown conclusions (1.28)-(1.37) of the main Theorem 1.1. We now complete the proof of Theorem 1.1 by establishing properties (1.39) and (1.40) as well.

Proposition 5.6 (Properties (1.39) and (1.40) of Theorem 1.1). Assume the approximating property (1.38), rewritten here as

$$
\begin{aligned}
& \left(\widehat{A}^{*}\right)^{\theta}\left(\widehat{A}_{h}^{*-1}\right)^{\theta} \in \mathscr{L}\left(V_{h} ; H\right), \quad \text { or } \\
& \left\|\left(\widehat{A}^{*}\right)^{\theta} x_{h}\right\|_{H} \leq C_{\theta}\left\|\left(\widehat{A}_{h}^{*}\right)^{\theta} x_{h}\right\|_{H}, \quad 0 \leq \theta<1, \forall x_{h} \in V_{h} .
\end{aligned}
$$

Then

(5.11)(i) $\left\|\left(\widehat{A}^{*}\right)^{\theta}\left(P_{h} \Pi_{h}-P\right) x\right\|_{H} \rightarrow 0$ as $h \downarrow 0, x \in H, 0 \leq \theta<1$;

(5.12)(ii) $\left\|\left(\hat{A}^{*}\right)^{\theta}\left(P_{h} \Pi_{h}-P\right) \hat{A}^{\theta} x\right\|_{H} \rightarrow 0$ as $h \downarrow 0, x \in H, 0 \leq \theta<\frac{1}{2}$.

The proof is given in the Supplement. After this, the proof of the main Theorem 1.1 is complete.

Completion of the proof of Theorem 1.2 is given in the Supplement.

\section{EXAMPLES}

In this section, we illustrate the applicability of Theorems 1.1 and 1.2 to some partial differential equations problems which exhibit the properties required by the theory. A few canonical cases of diffusion/heat equations with boundary control and strongly damped plate equations with point or boundary controls will be treated. For lack of space we shall concentrate only on the three most representative examples, which exemplify the following situations:

(i) $\gamma>\frac{1}{2}$ (heat equation with Dirichlet boundary conditions);

(ii) $\gamma>\frac{1}{2}$ and noncoercive nature of the problem (damped plate equation with point control);

(iii) $\gamma<\frac{1}{2}$ and $R(\lambda, A)$ noncompact (Kelvin-Voigt plate model with point control).

Other examples, as the ones given in [19], can be treated in the same manner.

6.1. Heat equation with Dirichlet boundary control. This problem has been considered in [18]. For the sake of completeness we shall show how it fits into the present theory.

6.1.1. Continuous problem. Let $\Omega \subset R^{n}$ be an open bounded domain with sufficiently smooth boundary $\Gamma$. In $\Omega$, we consider the Dirichlet mixed problem for the heat equation in the unknown $y(t, x)$ :

$$
\begin{array}{ll}
y_{t}=\Delta y+c^{2} y & \text { in }(0, T] \times \Omega \equiv Q, \\
y(0, \cdot)=y_{0} & \text { in } \Omega, \\
\left.y\right|_{\Sigma}=u & \text { in }(0, T] \times \Gamma \equiv \Sigma,
\end{array}
$$


with boundary control $u \in L_{2}(\Sigma)$ and $y_{0} \in L_{2}(\Omega)$. The cost functional which we wish to minimize is

$$
J(u, y)=\int_{0}^{\infty}\left\{\|y(t)\|_{L_{2}(\Omega)}^{2}+\|u(t)\|_{L_{2}(\Gamma)}^{2}\right\} d t .
$$

Abstract setting (see [17]). To put problem (6.1), (6.2) into the abstract setting of the preceding sections, we introduce the following operators and spaces:

$$
\begin{gathered}
A h=\Delta h+c^{2} h ; \quad \mathscr{D}(A)=H^{2}(\Omega) \cap H_{0}^{1}(\Omega), \\
Z=H=L_{2}(\Omega) ; \quad U=L_{2}(\Gamma), \\
B u=-A D_{1} u ; \quad R=I,
\end{gathered}
$$

where $D_{1}$ (Dirichlet map) is defined by

$$
\begin{gathered}
h=D_{1} g \quad \text { iff } \quad\left(\Delta+c^{2}\right) h=0 \text { in } \Omega ;\left.\quad h\right|_{\Gamma}=g, \\
D_{1}: \text { continuous } L_{2}(\Gamma) \rightarrow H^{1 / 2}(\Omega) \subset H^{1 / 2-2 \varepsilon}(\Omega) \equiv \mathscr{D}\left(A_{D}^{1 / 4-\varepsilon}\right) \\
A_{D} h=-\Delta h, \quad \forall \varepsilon>0, \quad \mathscr{D}\left(A_{D}\right)=H^{2}(\Omega) \cap H_{0}^{1}(\Omega), \quad
\end{gathered}
$$

by elliptic theory, and where $A$ in (6.5) is the isomorphic extension of $A$ in (6.3), from, say, $L_{2}(\Omega) \rightarrow[\mathscr{D}(A)]^{\prime}$.

The approximation framework for problem (6.1) and the verification of all required assumptions for both the continuous as well as the discrete problem are given in the Supplement.

\subsection{Structurally damped plates with point control.}

6.2.1. Continuous problem. The case $\alpha=\frac{1}{2}[4,5]$. Consider the following model of a plate equation in the deflection $w(t, x)$, where $\rho>0$ is any constant:

$$
\begin{array}{ll}
w_{t t}+\Delta^{2} w-\rho \Delta w_{t}=\delta\left(x-x^{0}\right) u(t) & \text { in }(0, T] \times \Omega=Q, \\
w(0, \cdot)=w_{0} ; w_{t}(0, \cdot)=w_{1} . & \text { in } \Omega, \\
\left.\left.w\right|_{\Sigma} \equiv \Delta w\right|_{\Sigma} \equiv 0 & \text { in }(0, T] \times \Gamma=\Sigma,
\end{array}
$$

with load concentrated at the interior point $x^{0}$ of an open bounded (smooth) domain $\Omega$ of $R^{n}, n \leq 3$. Regularity results for problem (6.9), and other problems of this type, are given in [27]. Consistently, the cost functional we wish to minimize is

$$
J(u, w)=\int_{0}^{\infty}\left\{\|w(t)\|_{H^{2}(\Omega)}^{2}+\left\|w_{t}(t)\right\|_{L_{2}(\Omega)}^{2}+\|u(t)\|_{L_{2}(\Gamma)}^{2}\right\} d t,
$$

where $\left\{w_{0}, w_{1}\right\} \in\left[H^{2}(\Omega) \cap H_{0}^{1}(\Omega)\right] \times L_{2}(\Omega)$.

Abstract setting. To put problems (6.9), (6.10) into the abstract setting of the preceding sections, we introduce the strictly positive definite operator

$$
\mathscr{A} h=\Delta^{2} h ; \quad \mathscr{D}(\mathscr{A})=\left\{h \in H^{4}(\Omega):|h|_{\Gamma}=\left.\Delta h\right|_{\Gamma}=0\right\}
$$


and select the following spaces and operators:

$$
\begin{aligned}
H & \equiv \mathscr{D}\left(\mathscr{A}^{1 / 2}\right) \times L_{2}(\Omega)=\left[H^{2}(\Omega) \cap H_{0}^{1}(\Omega)\right] \times L_{2}(\Omega) ; \quad U=\mathbb{R}^{1}, \\
A & =\left[\begin{array}{cc}
0 & I \\
-\mathscr{A} & -\rho \mathscr{A}^{1 / 2}
\end{array}\right] ; \quad B u=\left[\begin{array}{c}
0 \\
\delta\left(x-x^{0}\right) u
\end{array}\right] ; \quad R=I
\end{aligned}
$$

to obtain the abstract model (1.1), (1.2). Again, approximation framework and verification of all required assumptions are given in the Supplement.

\subsection{Kelvin-Voigt plate equation with point control.}

6.3.1. Continuous problem. The case $\alpha=1$ [4, 5]. The Kelvin-Voigt model for a plate equation in the deflection $w(t, x)$ is

$$
\begin{array}{lll}
(6.14 \mathrm{a}) & w_{t t}+\Delta^{2} w+\rho \Delta^{2} w_{t}=\delta\left(x-x^{0}\right) u(t) & \text { in }(0, T] \times \Omega=Q \\
(6.14 \mathrm{~b}) & w(0, \cdot)=w_{0} ; w_{t}(0, \cdot)=w_{1} & \text { in } \Omega, \\
(6.14 \mathrm{c}) & \left.\Delta w\right|_{\Sigma}+(1-\mu) B_{1} w \equiv 0 & \text { in }(0, T] \times \Gamma=\Sigma, \\
(6.14 \mathrm{~d}) & \left.\frac{\partial \Delta w}{\partial \nu}\right|_{\Sigma}+(1-\mu) B_{2} w \equiv 0 & \text { in } \Sigma,
\end{array}
$$

with $0<\mu<\frac{1}{2}$ the Poisson modulus and $\rho>0$ any constant, where again $x^{0}$ is an interior point of the open bounded $\Omega \subset R^{n}, n \leq 2$. The boundary operators $B_{1}$ and $B_{2}$ are zero for $n=1$, and for $n=2$ [16]:

$$
\begin{aligned}
& B_{1} w=2 \nu_{1} \nu_{2} w_{x y}-\nu_{1}^{2} w_{y y}-\nu_{2}^{2} w_{x x} ; \\
& B_{2} w=\frac{\partial}{\partial \tau}\left[\left(\nu_{1}^{2}-\nu_{2}^{2}\right) w_{x y}+\nu_{1} \nu_{2}\left(w_{y y}-w_{x x}\right)\right],
\end{aligned}
$$

where $\partial / \partial \tau$ is the tangential derivative. Regularity results for problem (6.14) are given in [27]. Consistently with these, we take the cost functional to be the same as $(6.10)$ with $\left\{w_{0}, w_{1}\right\} \in H^{2}(\Omega) \times L_{2}(\Omega)$.

Abstract setting. We introduce the nonnegative self-adjoint operator

$$
\mathscr{A} h=\Delta^{2} h,
$$

$$
\mathscr{D}(\mathscr{A})=\left\{h \in H^{4}(\Omega): \Delta h+\left.(1-\mu) B_{1} h\right|_{\Gamma}=0 ; \frac{\partial \Delta h}{\partial \nu}+\left.(1-\mu) B_{2} h\right|_{\Gamma}=0\right\},
$$

and select the following spaces and operators:

$$
\begin{gathered}
H=\mathscr{D}\left(\mathscr{A}^{1 / 2}\right) \times L_{2}(\Omega)=H^{2}(\Omega) \times L_{2}(\Omega) ; \quad U=\mathbb{R}^{1}, \\
A=\left[\begin{array}{cc}
0 & I \\
-\mathscr{A} & -\rho \mathscr{A}
\end{array}\right] ; \quad B u=\left[\begin{array}{c}
0 \\
\delta\left(x-x^{0}\right) u
\end{array}\right] ; \quad R=I
\end{gathered}
$$

to obtain the abstract model (1.1), (1.2). Approximation framework as well as verification of all required assumptions are given in the Supplement. 


\section{BIBLIOGRAPHY}

1. H. T. Banks and K. Kunisch, The linear regulator problem for parabolic systems, SIAM J. Control Optim. 22 (1984), 684-699.

2. I. Babuška and A. Aziz, The mathematical foundations of the finite element method with applications to partial differential equations, Academic Press, New York, 1972.

3. J. H. Bramble, A. H. Schatz, V. Thomée, and L. B. Wahlbin, Some convergence estimates for semidiscrete Galerkin type approximations for parabolic equations, SIAM J. Numer. Anal. 14 (1977), 218-241.

4. S. Chen and R. Triggiani, Proof of two conjectures of G. Chen and D. L. Russell on structural damping for elastic systems: The case $\alpha=1 / 2$, Proc. Seminar in Approximation and Optimization (University of Havana, Cuba, January 12-14, 1987), Lecture Notes in Math., vol. 1354, Springer-Verlag, 1988, pp. 234-256.

5. __ Proof of extensions of two conjectures on structural damping for elastic systems, Pacific J. Math. 136 (1989), 15-55.

6. __ Gevrey class semigroups arising from elastic systems with gentle dissipation: The case $0<\alpha<1 / 2$, Proc. Amer. Math. Soc. 110 (1990), 401-415.

7. __ Characterization of domains of fractional powers of certain operators arising in elastic systems, and applications, J. Differential Equations 88 (1990), 279-293.

8. G. DaPrato and A. Ichikawa, Riccati equations with unbounded coefficients, Ann. Mat. Pura Appl. 140 (1985), 209-221.

9. F. Flandoli, Algebraic Riccati equations arising in boundary control problems, SIAM J. Control Optim. 25 (1987), 612-636.

10. _ Riccati equation arising in a boundary control problem with distributed parameters, SIAM J. Control Optim. 22 (1984), 76-86.

11. J. S. Gibson, The Riccati integral equations for optimal control problems on Hilbert spaces, SIAM J. Control Optim. 17 (1979), 537-565.

12. J. Gardiner and A. Laub, Matrix sign function implementations on a hypercube multiprocessor, Proc. CDC Conf., December 1988, pp. 1466-1471.

13. T. Kato, Perturbation theory of linear operators, Springer-Verlag, New York, 1966.

14. I. Lasiecka, Convergence estimates for semidiscrete approximations of nonselfadjoint parabolic equations, SIAM J. Numer. Anal. 21 (1984).

15. __ Galerkin approximations of abstract parabolic boundary value problems with rough boundary data; $L_{p}$ theory, Math. Comp. 47 (1986), 55-75.

16. J. Lagnese, Boundary stabilization of thin plates, SIAM Stud. Appl. Math., vol. 10, SIAM, Philadelphia, PA, 1989.

17. I. Lasiecka and R. Triggiani, The regulator problem for parabolic equations with Dirichlet boundary control. I, Appl. Math. Optim. 16 (1987), 147-168.

18. _ The regulator problem for parabolic equations with Dirichlet boundary control. II, Appl. Math. Optim. 16 (1987), 187-216.

19. __ Algebraic Riccati equations with applications to boundary/point control problems: Continuous theory and approximation theory, Perspectives in Control Theory (B. Jakubczyk, K. Malanowski, and W. Respondek, eds.), Birkhäuser, 1990, pp. 175-235; Expanded version to appear as a volume in Springer Verlag Lecture Notes in Control and Information Sciences.

20. __ Dirichlet boundary control problem for parabolic equations with quadratic cost: Analyticity and Riccati's feedback synthesis, SIAM J. Control Optim. 21 (1983), 41-67.

21. A. Manitius and R. Triggiani, Function space controllability of linear retarded systems: $A$ derivation from abstract operator conditions, SIAM J. Control Optim. 16 (1978), 599-645.

22. A. Pazy, Semigroups of operators and applications to partial differential equations, SpringerVerlag, 1983.

23. C. Sadosky, Interpolation of operators and singular integrals, Marcel Dekker, New York, 1979. 
24. V. Thomée, Galerkin finite element methods for parabolic problems, Lecture Notes in Math., vol. 1054, Springer, Berlin, 1984.

25. R. Triggiani, On the stabilizability problem in Banach space, J. Math. Anal. Appl. 52 (1975), 383-403.

26. __ Regularity of structurally damped systems with point/boundary control, preprint 1989; J. Math. Anal. Appl. (to appear).

27. __ Boundary feedback stabilization of parabolic equations, Appl. Math. Optim. 6 (1980), 201-220.

28. H. Triebel, Interpolation theory, function spaces, differential operators, VEB, North-Holland, 1978.

Department of Applied Mathematics, University of Virginia, Charlottesville, VirGINIA 22903-2442 
MATHEMATICS OF COMPUTATION

VOLUME 57, NUMBER 196

OCTOBER 1991, PAGES S13-S37

\title{
Supplement to
}

\section{NUMERICAL APPROXIMATIONS OF ALGEBRAIC RICCATI EQUATIONS FOR ABSTRACT SYSTEMS MODELLED BY ANALYTIC SEMIGROUPS, AND APPLICATIONS}

\author{
I. LASIECKA AND R. TRIGGIANI
}

\section{Table of Contents of Supplement}

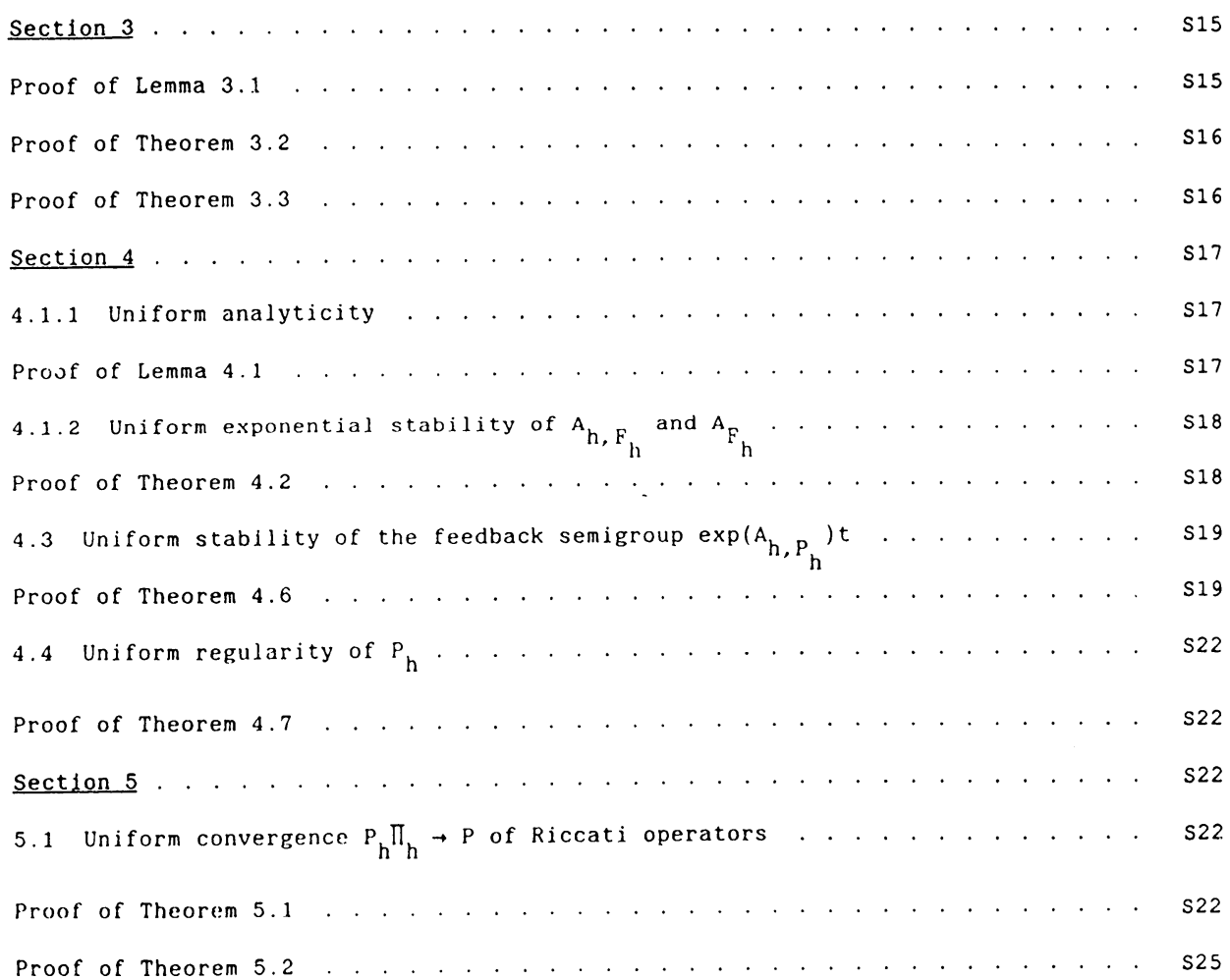


5.2 Uniform convergence $\mathrm{B}_{h}{ }^{*} \mathrm{P}_{\mathrm{h}} \Pi_{\mathrm{h}} \rightarrow \mathrm{B}^{*} \mathrm{P}$ of gain operaturs . . . . . . . . . . . . . S25

Proof of Theorem 5.3 . . . . . . . . . . . . . . . . . . . . . . . . . . S25

Proof of Lemma 5.4 . . . . . . . . . . . . . . . . . . . . . . . . . . . . . . 526

5.3 Uniform convergence $u_{h}^{0} \rightarrow u^{0}$. . . . . . . . . . . . . . . . . . . . . . . . . s27

Proof of Corollary 5.5 . . . . . . . . . . . . . . . . . . . . . . . . . . . . . S27

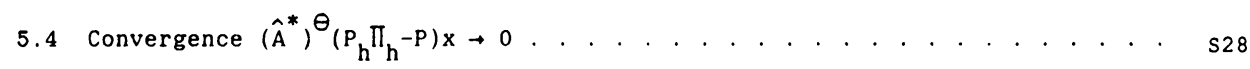

Proof of Proposition 5.6 . . . . . . . . . . . . . . . . . . . . . . . . . . . . s28

5.5 Completion of the proof of main Theorem 1.2 . . . . . . . . . . . . . . . . S28

section 6. . . . . . . . . . . . . . . . . . . . . . . . . . . . . . . . . s29

Example 6.1: Heat equation with Dirichlet boundary control . . . . . . . . . . . S29

Example 6.2: Structurally damped plates with point control . . . . . . . . . . . S31

Example 6.3: Kelvin-Voigt plate equation with point control.......... . . S35 

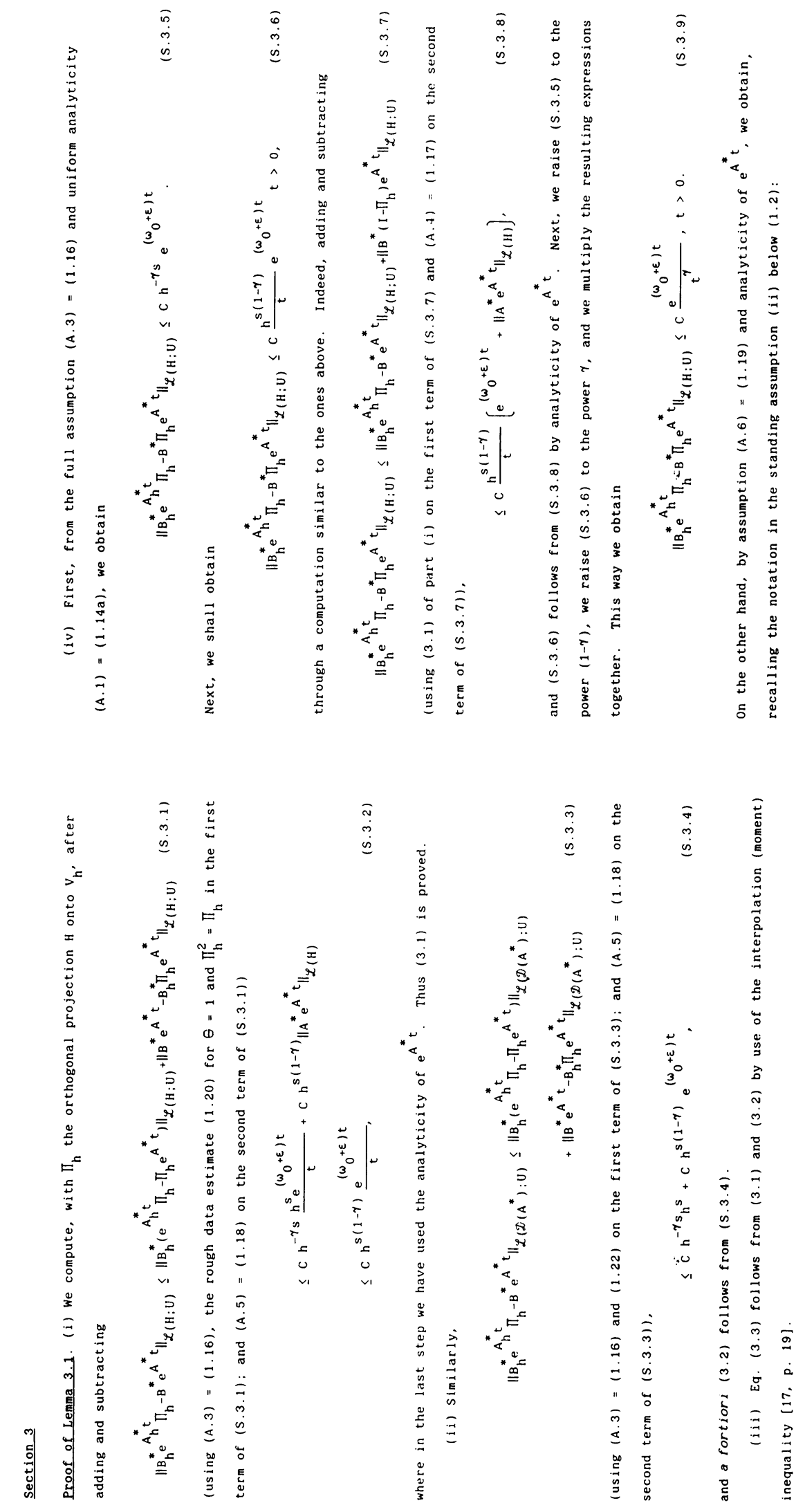

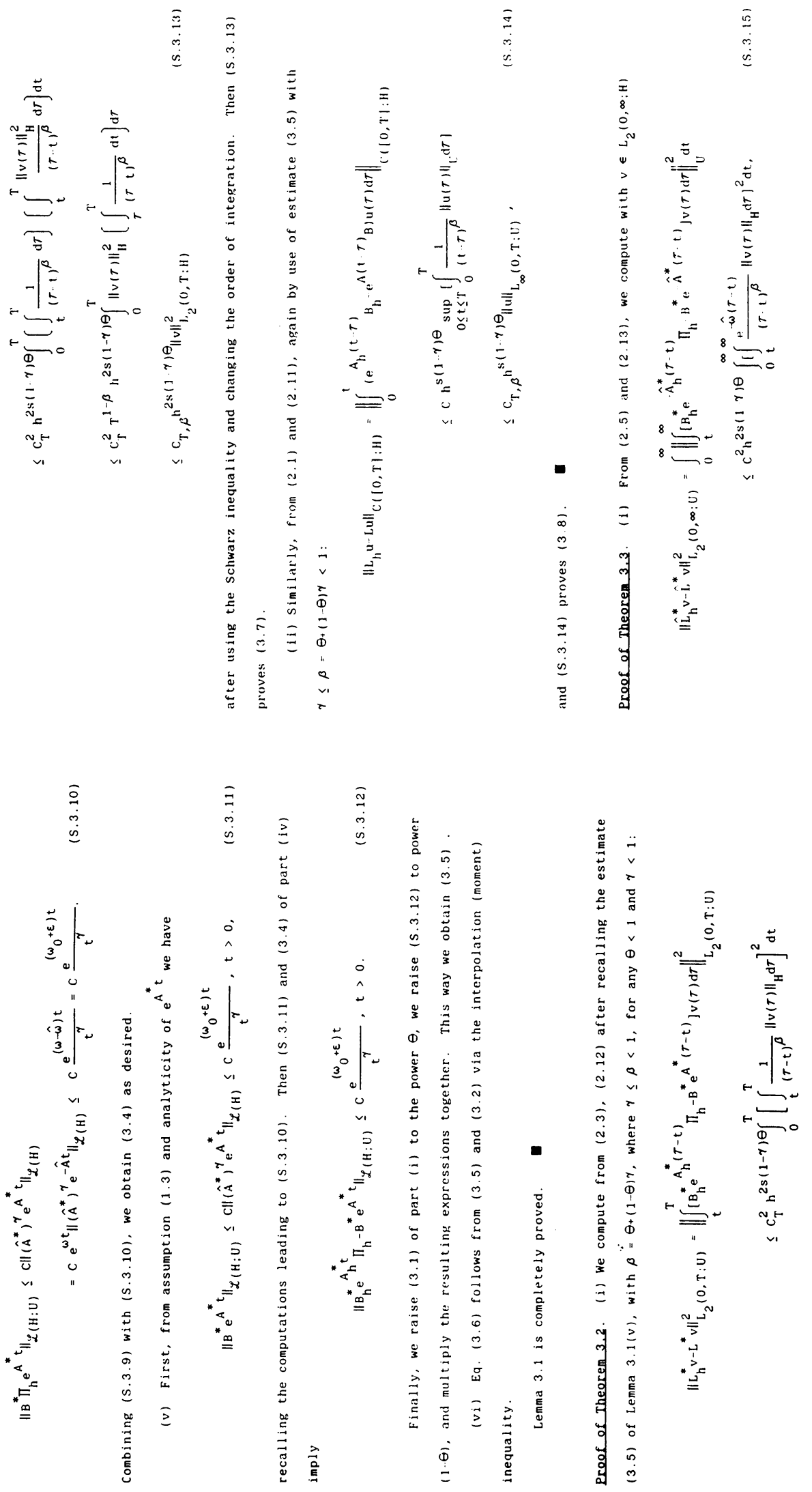

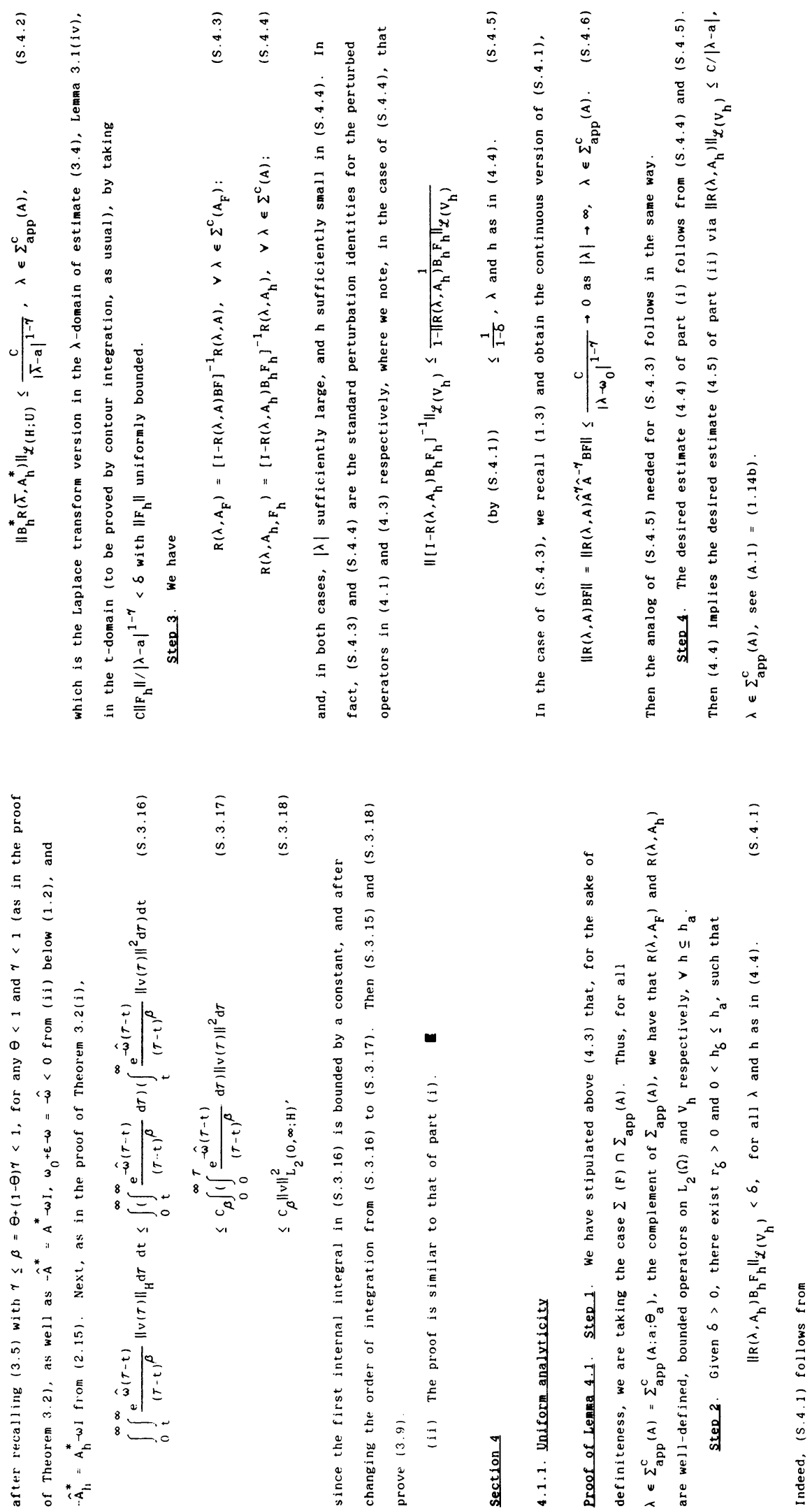

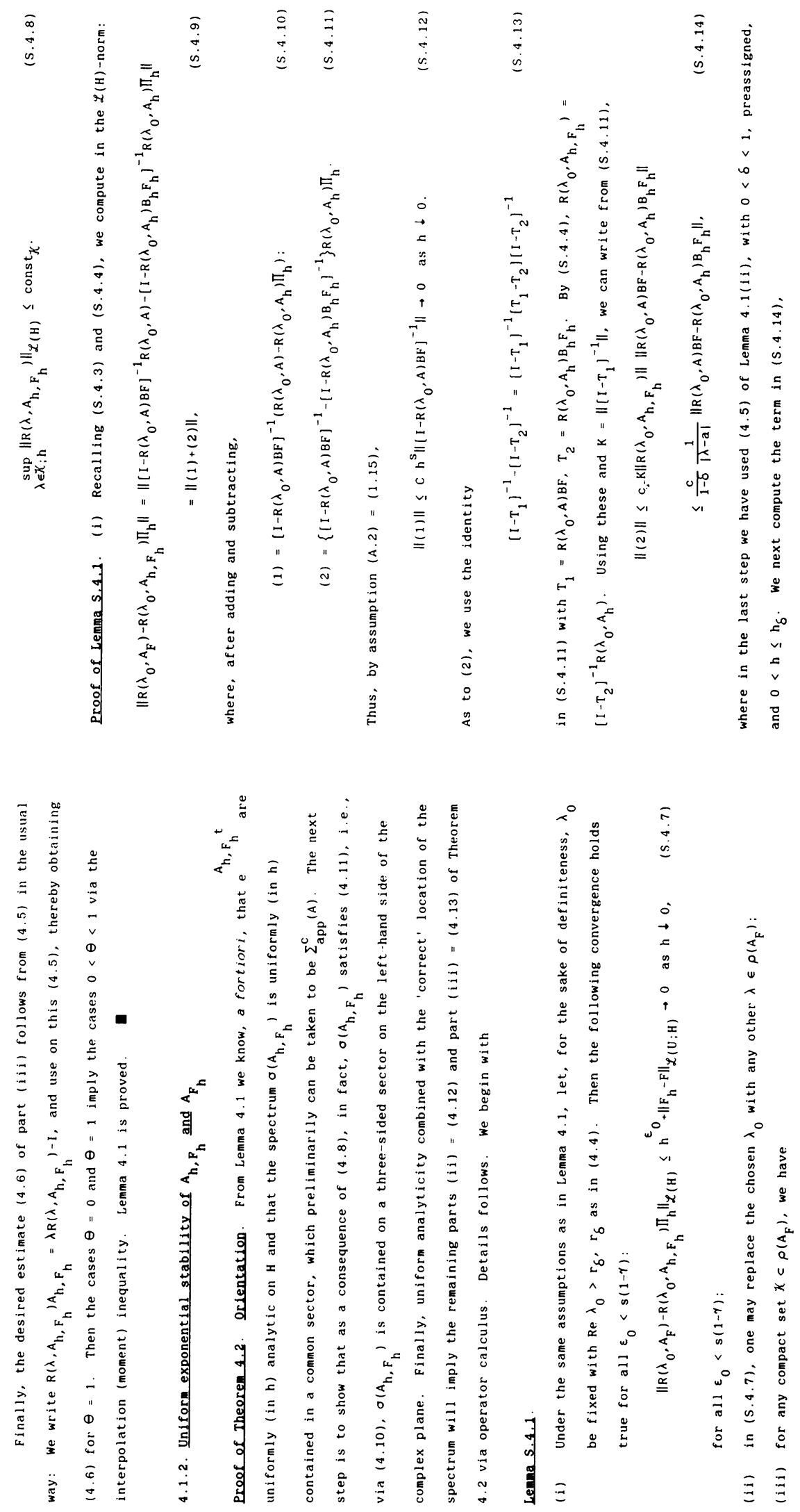


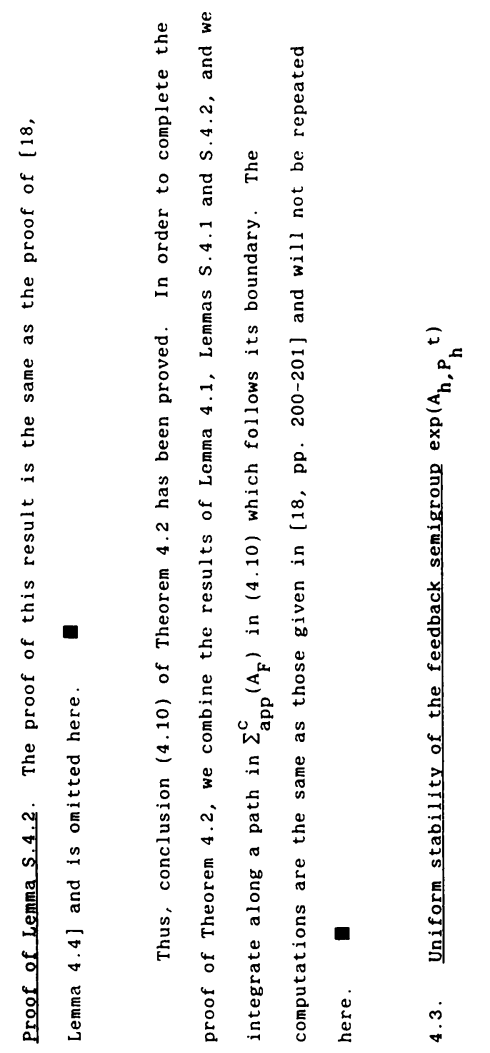

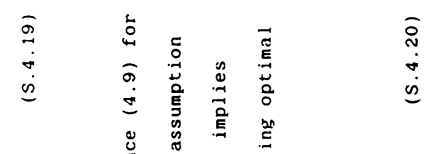


SUPPLEMENT
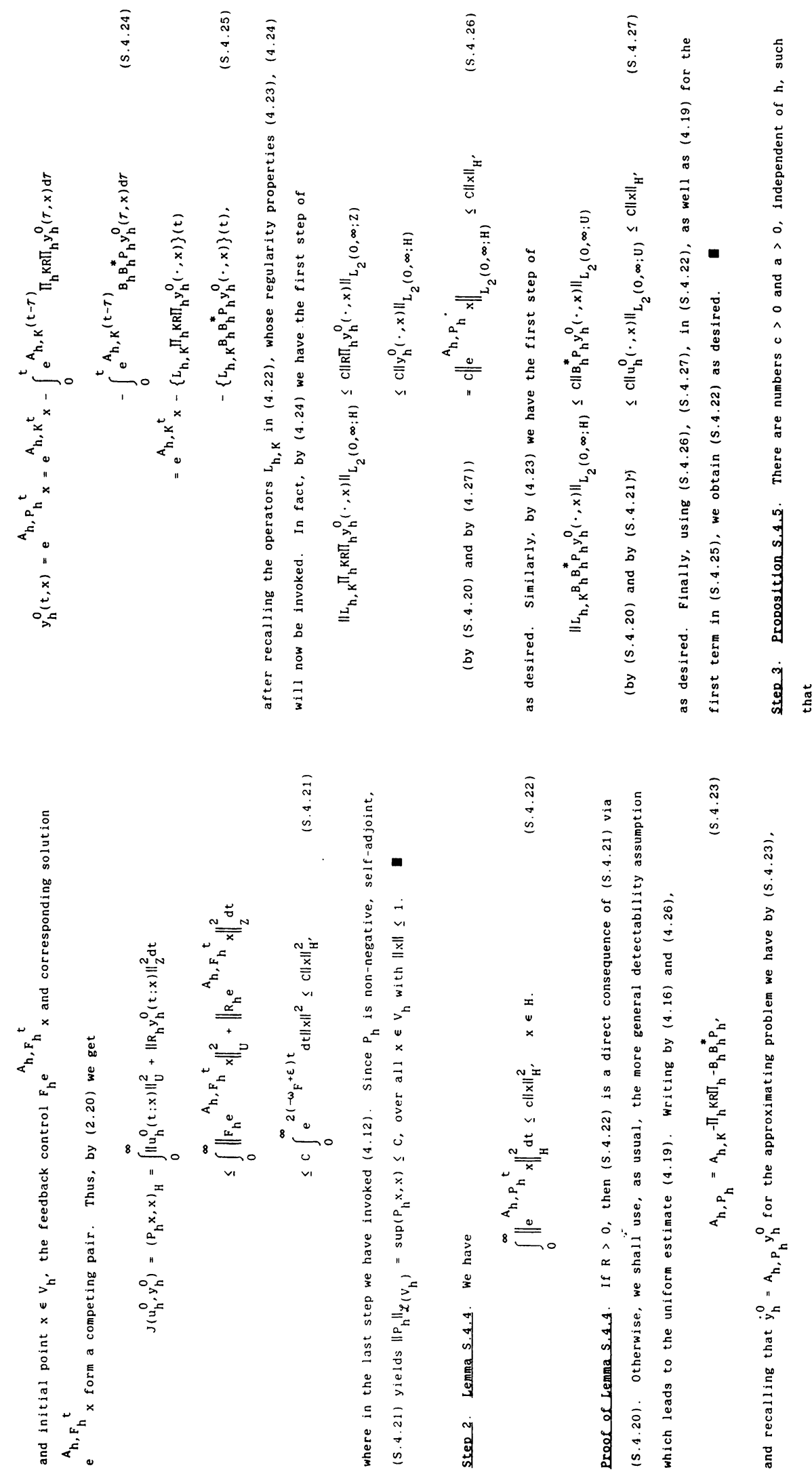

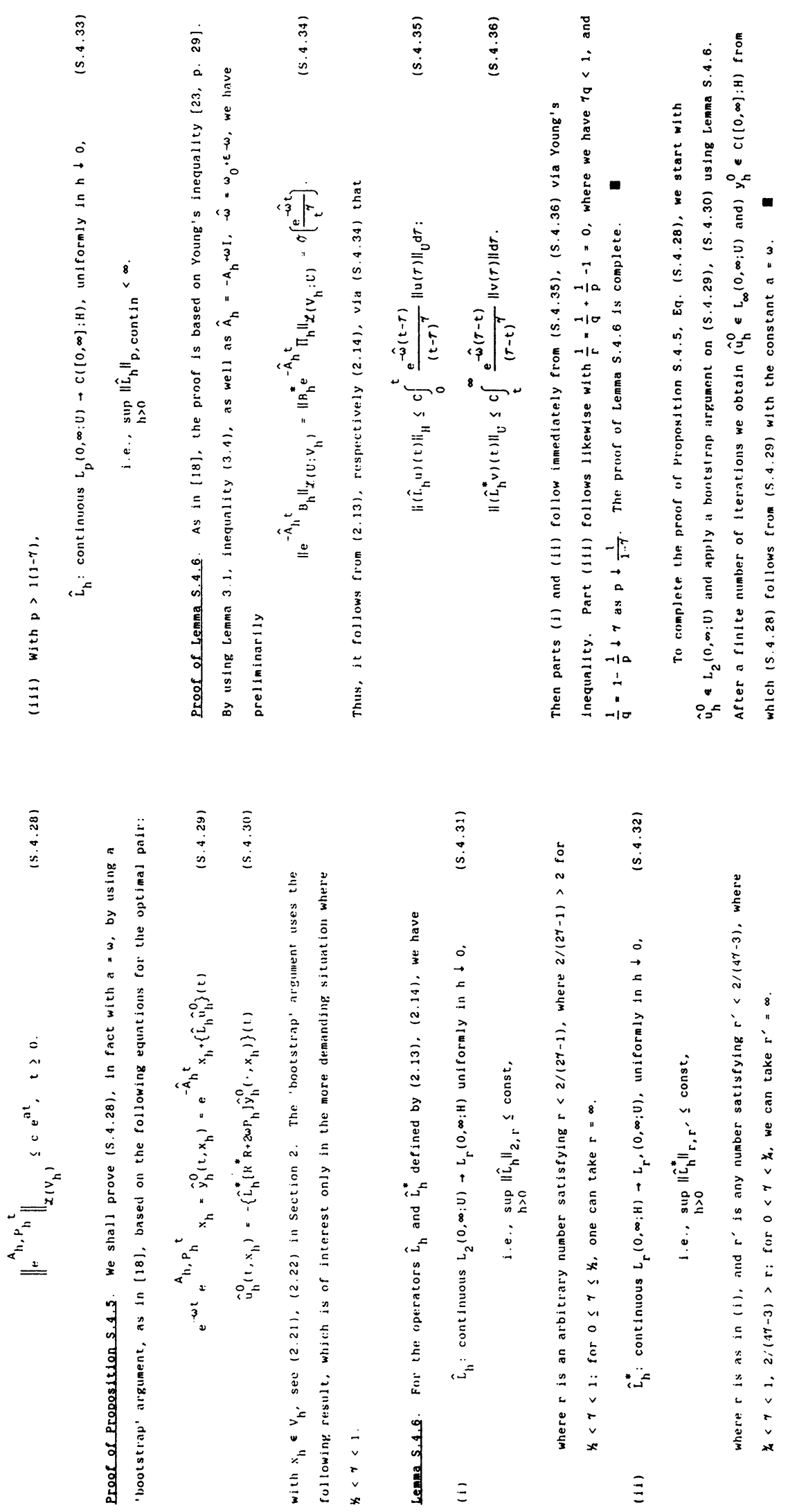

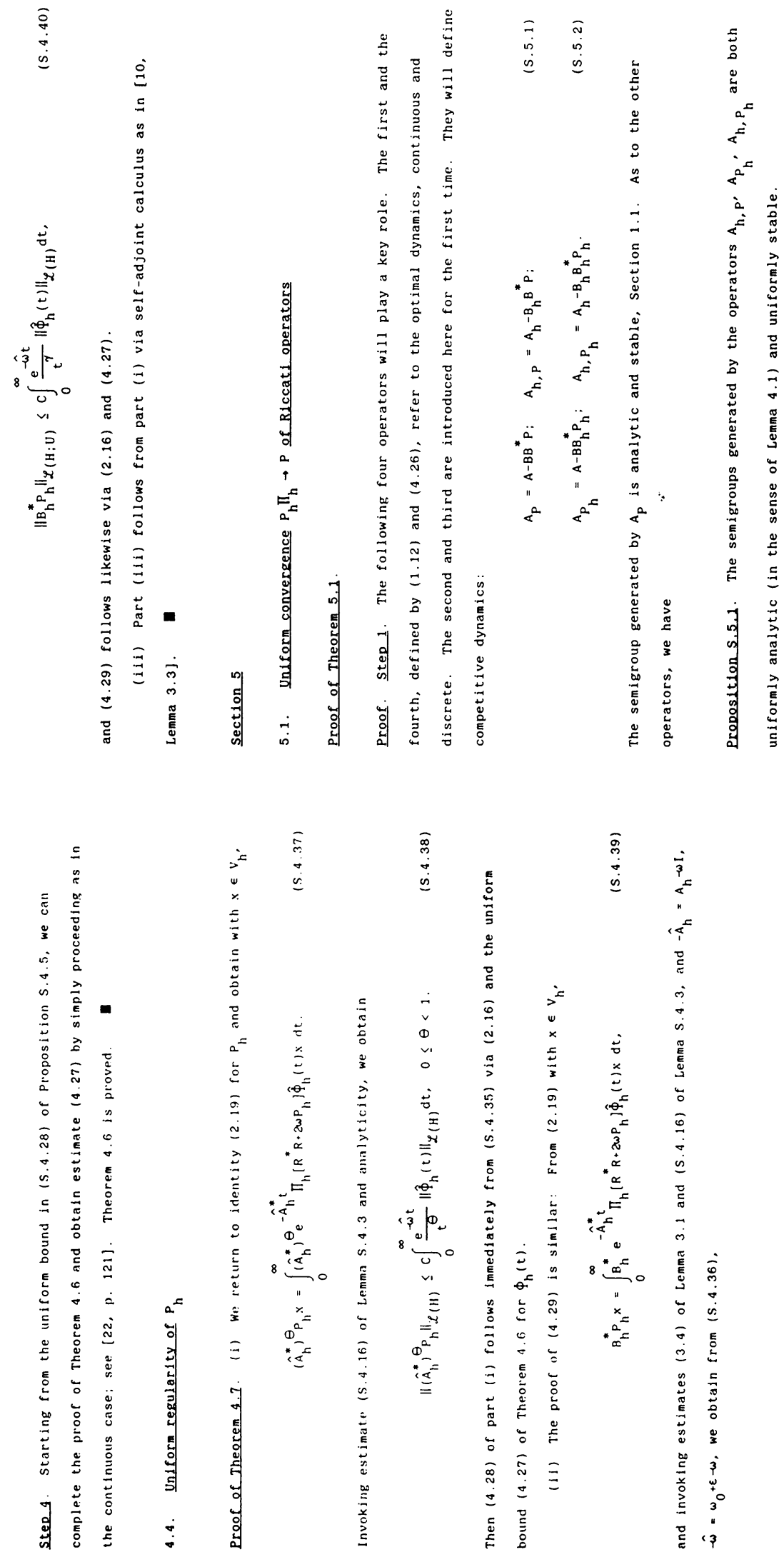

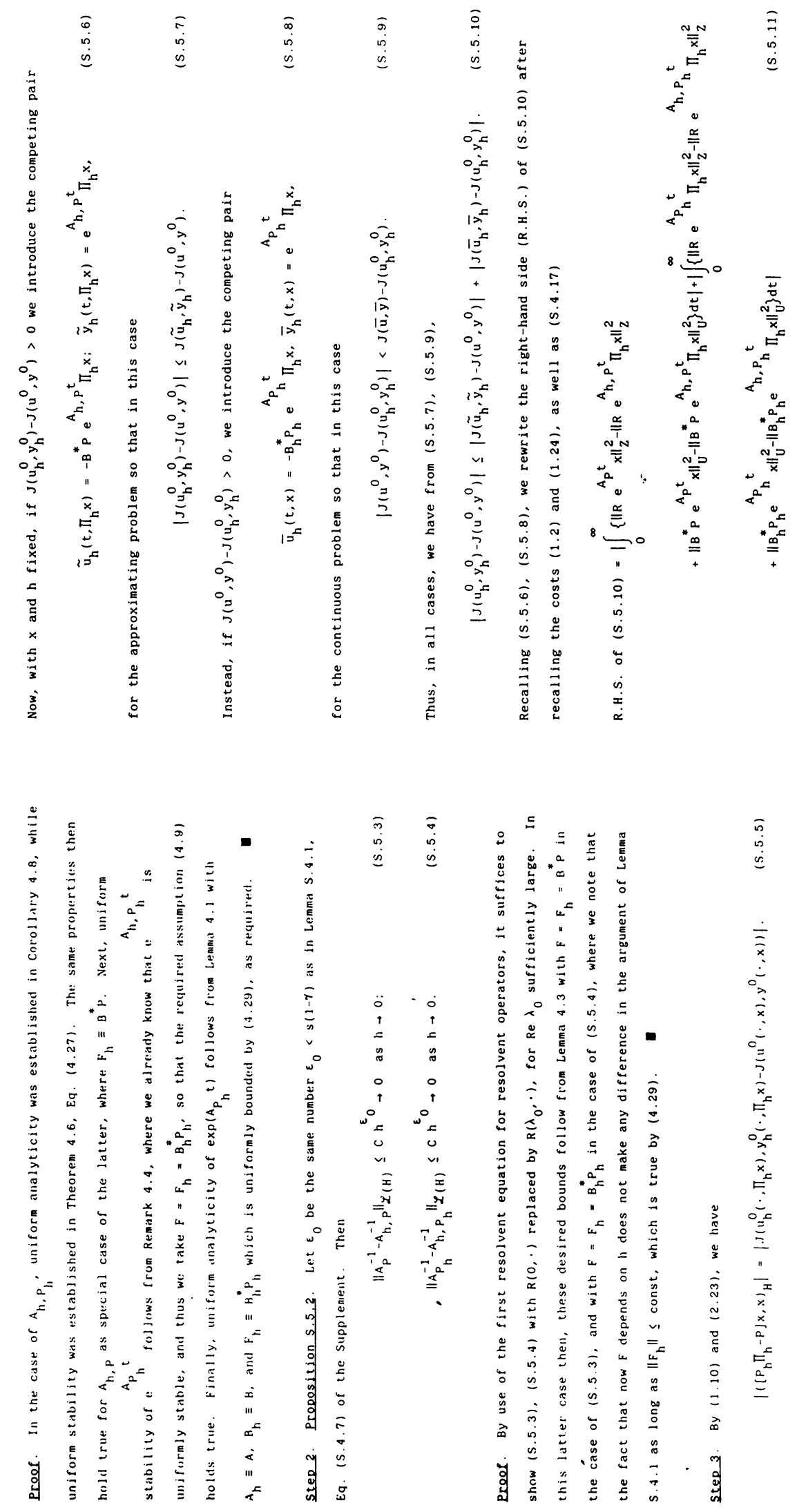

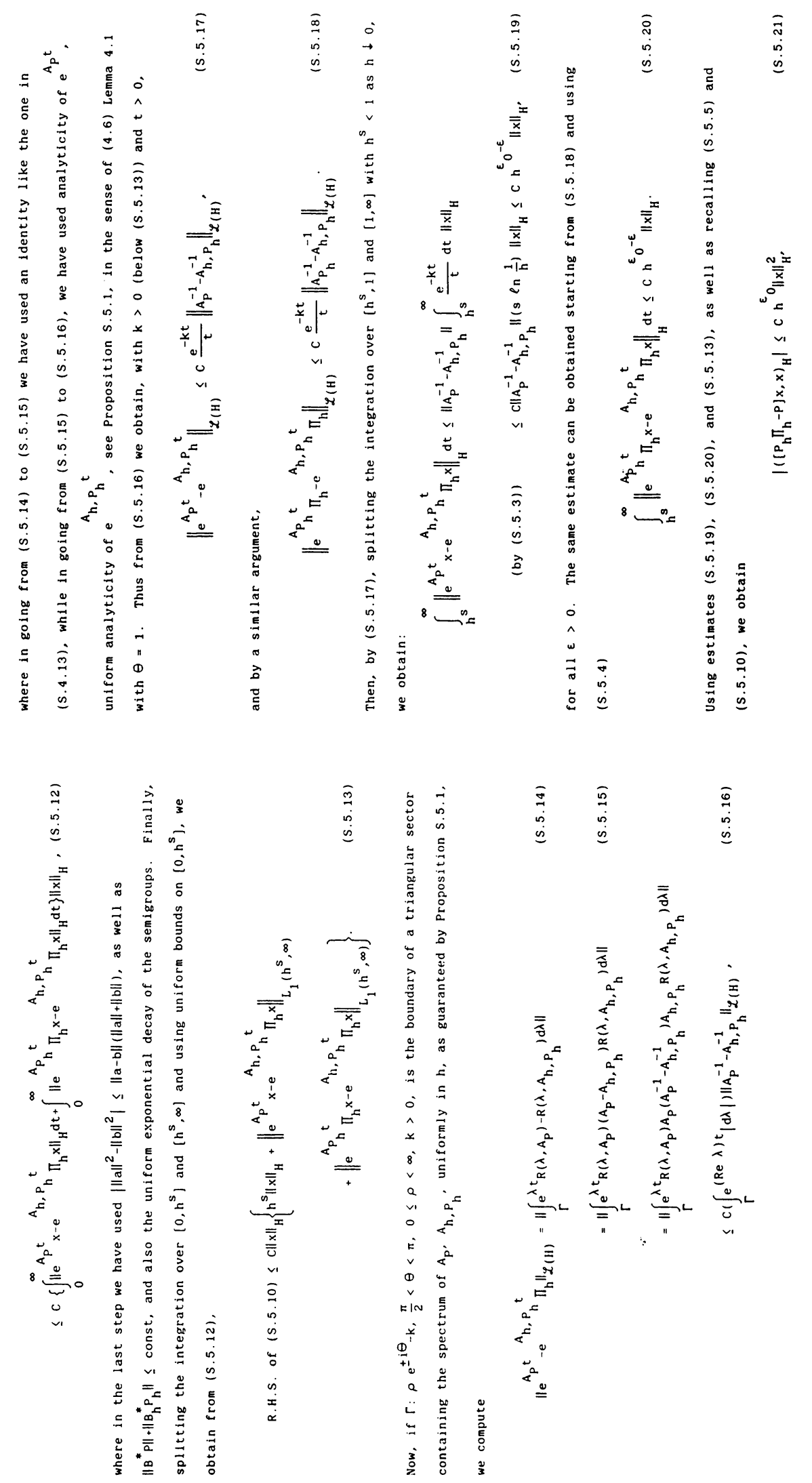

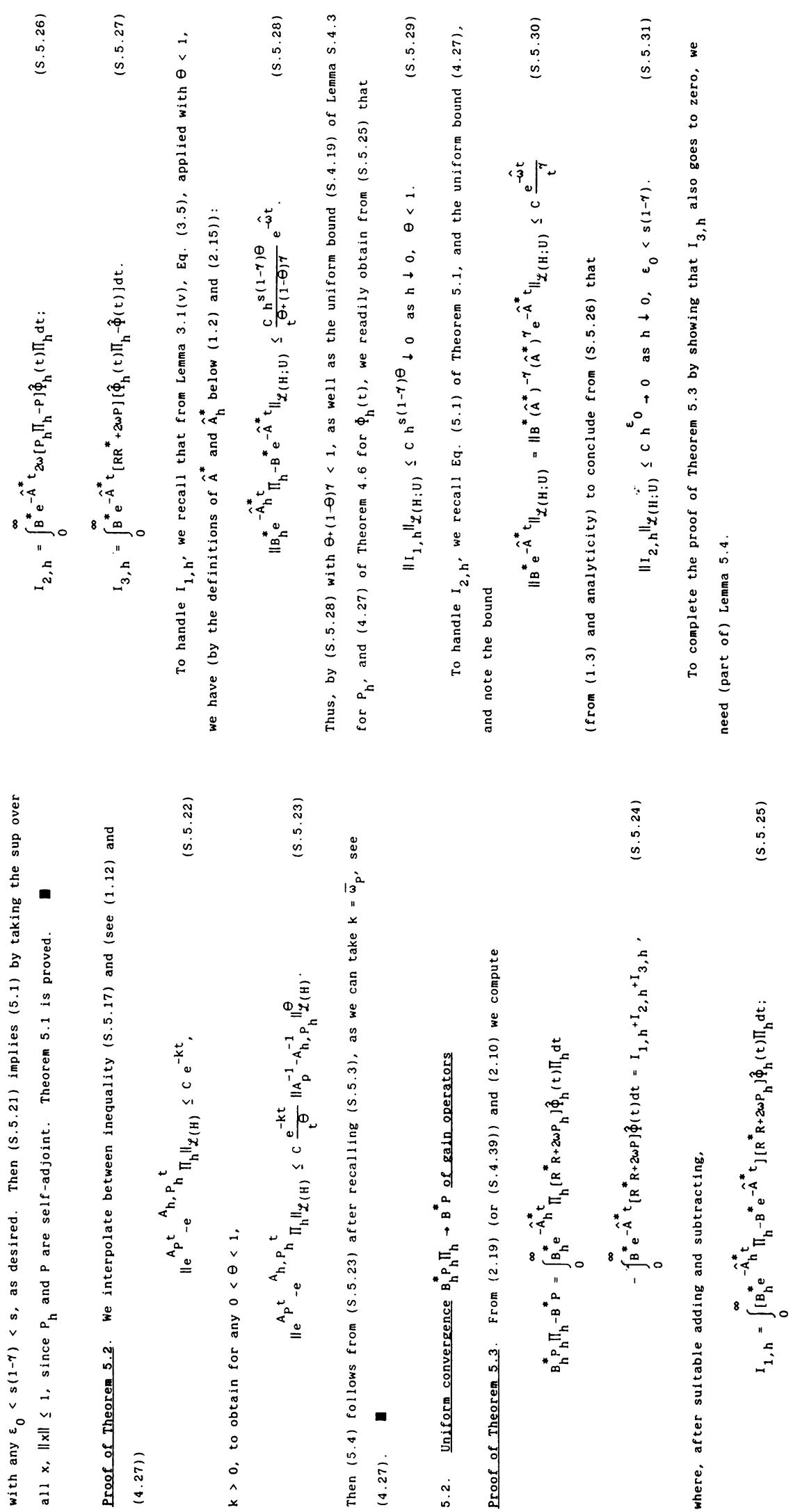

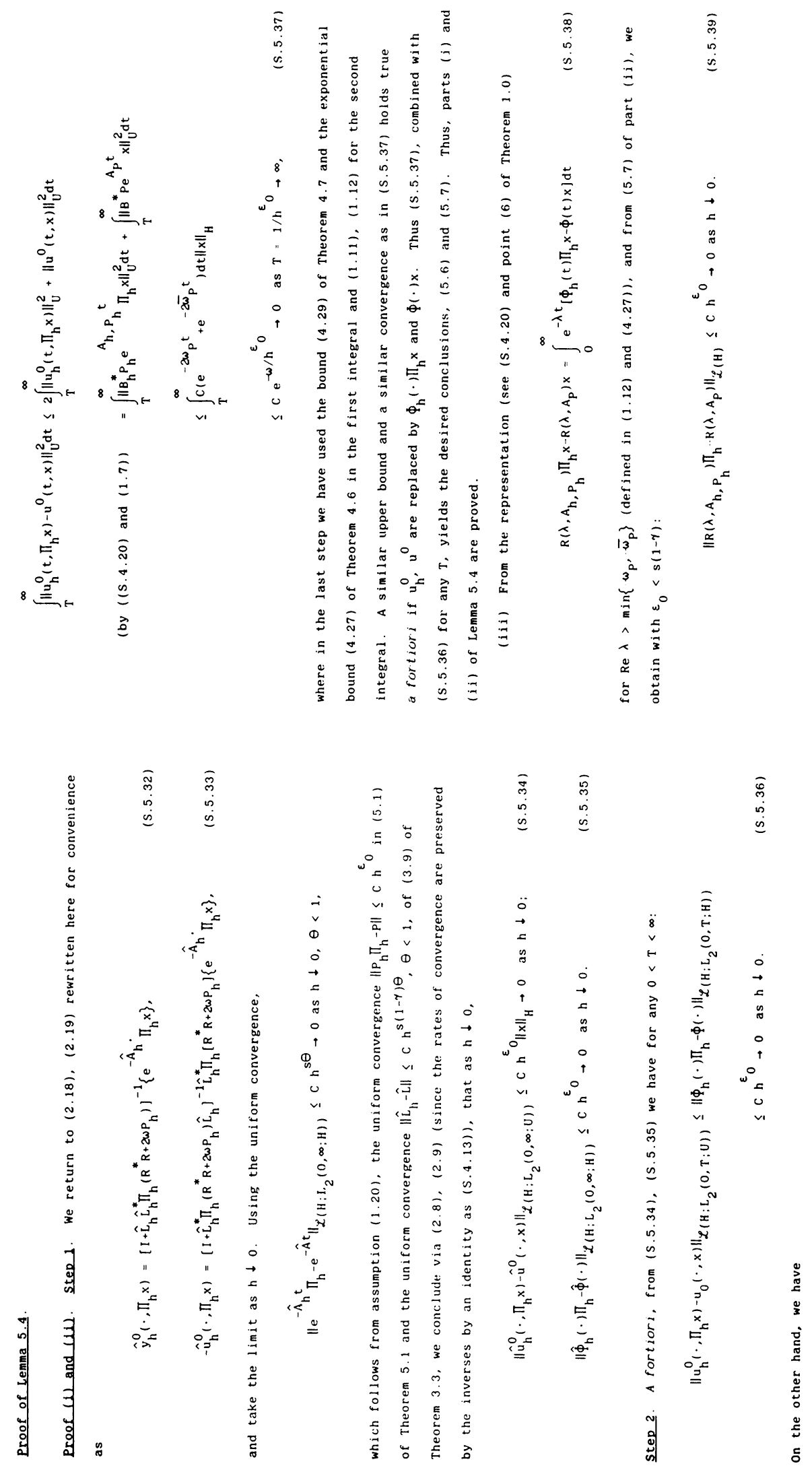

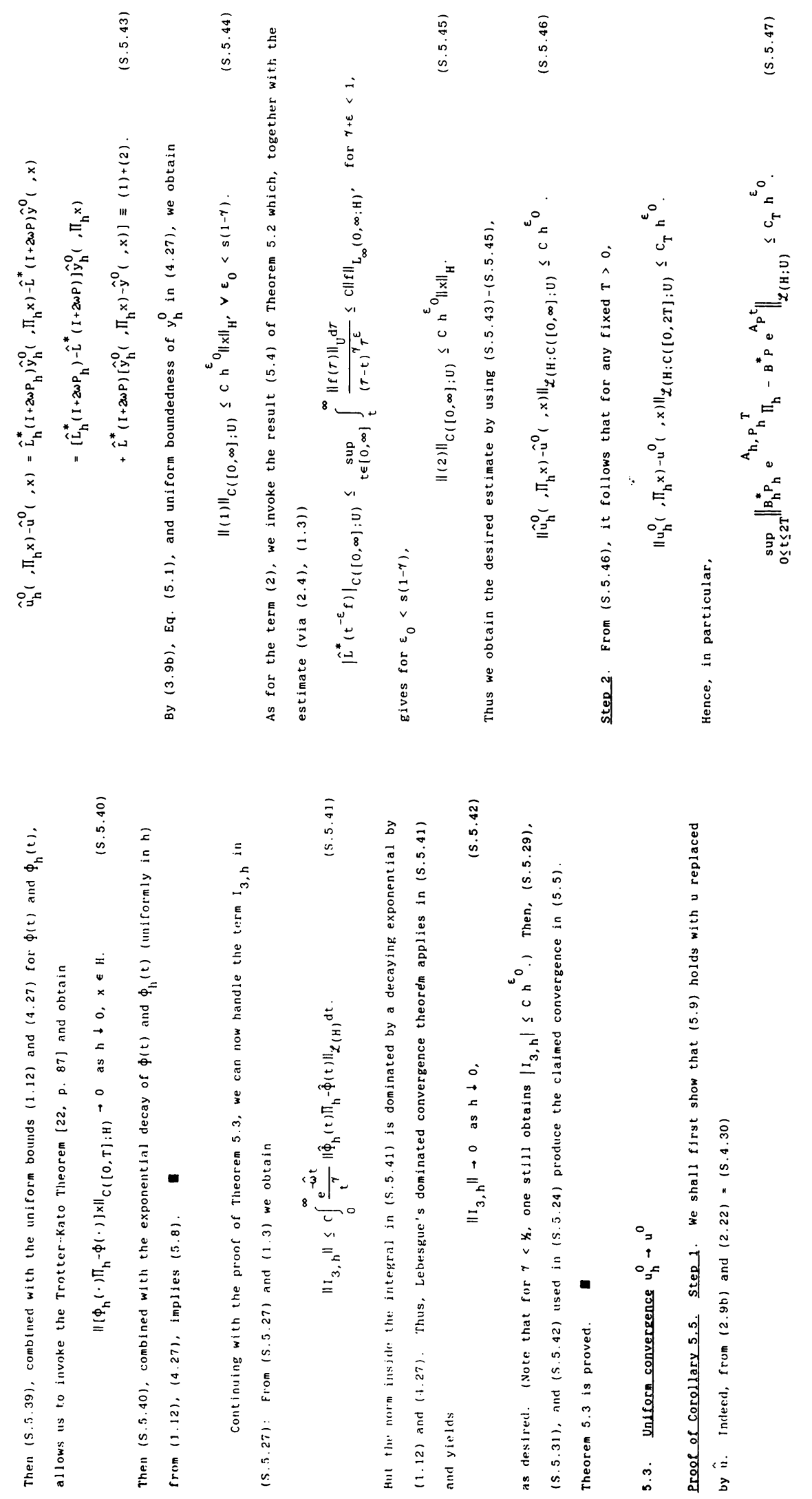

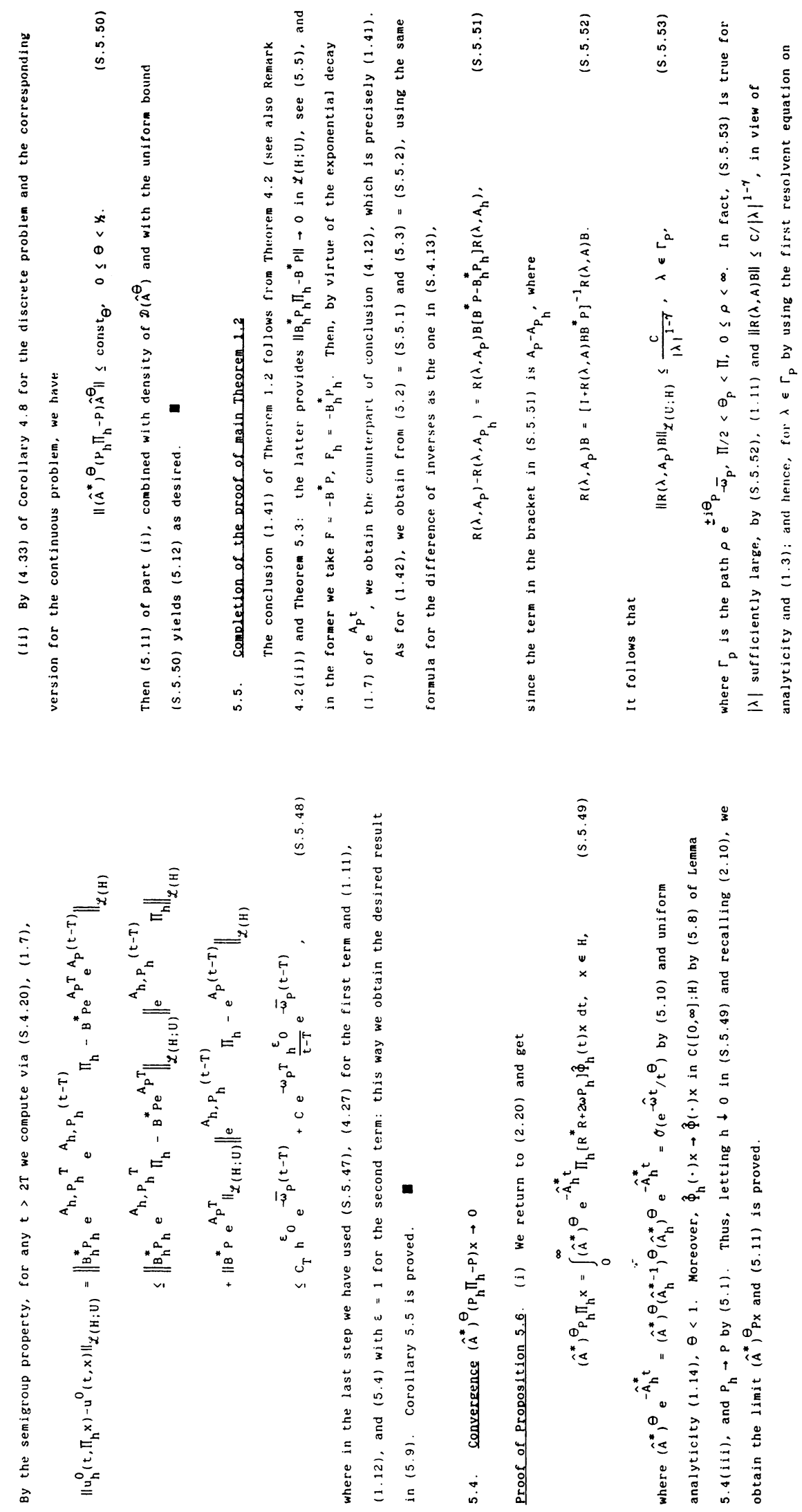

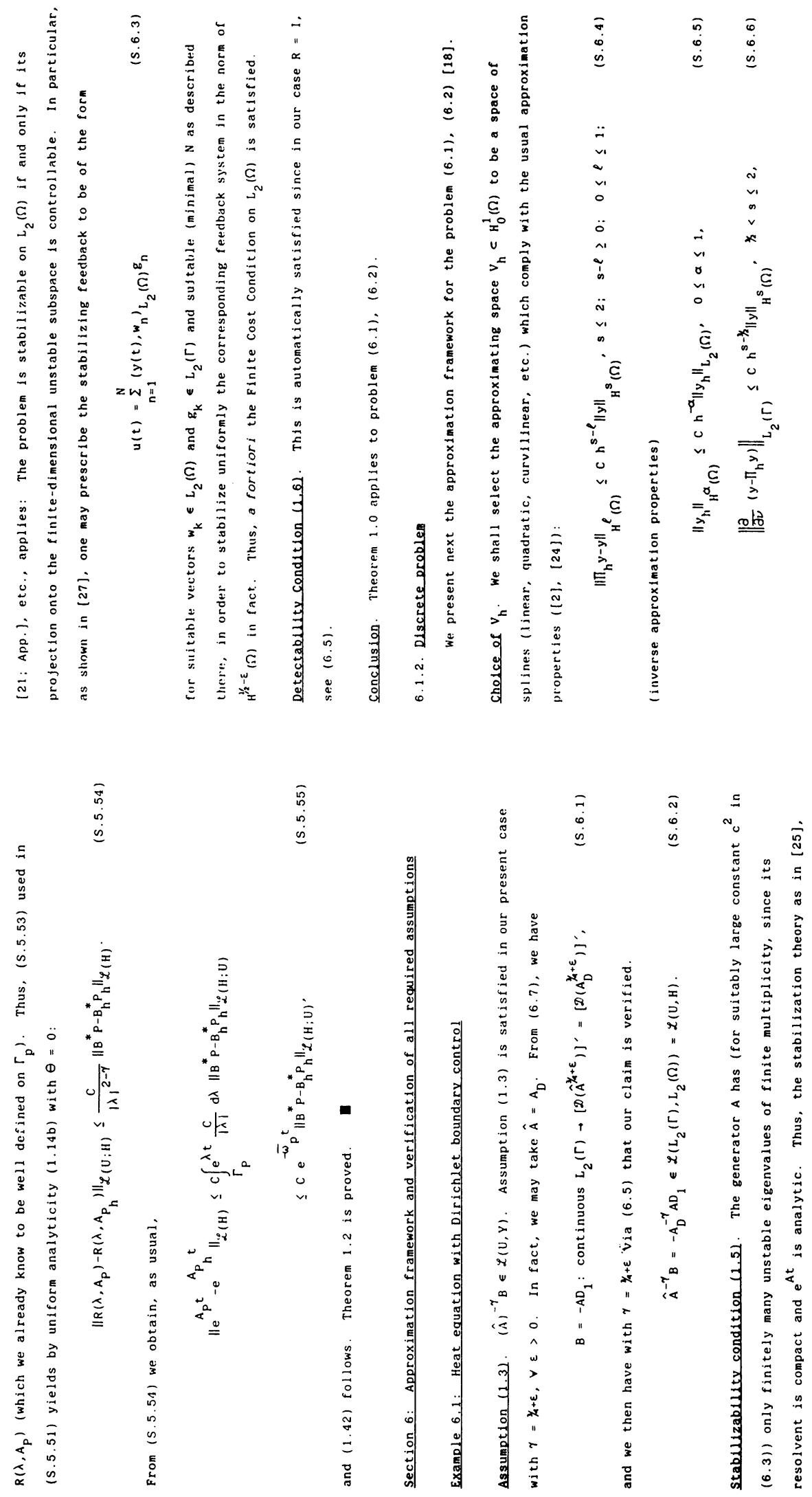


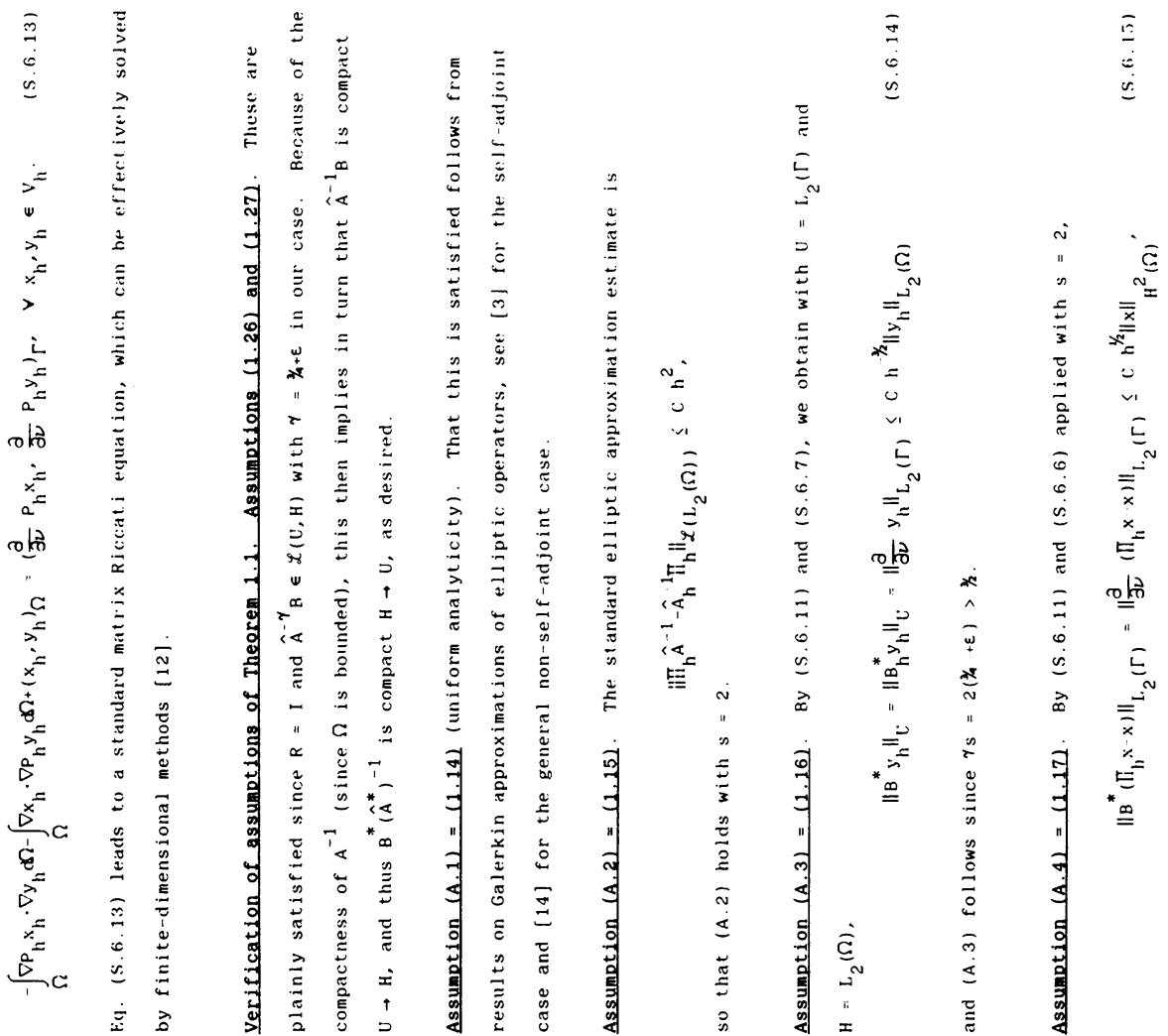

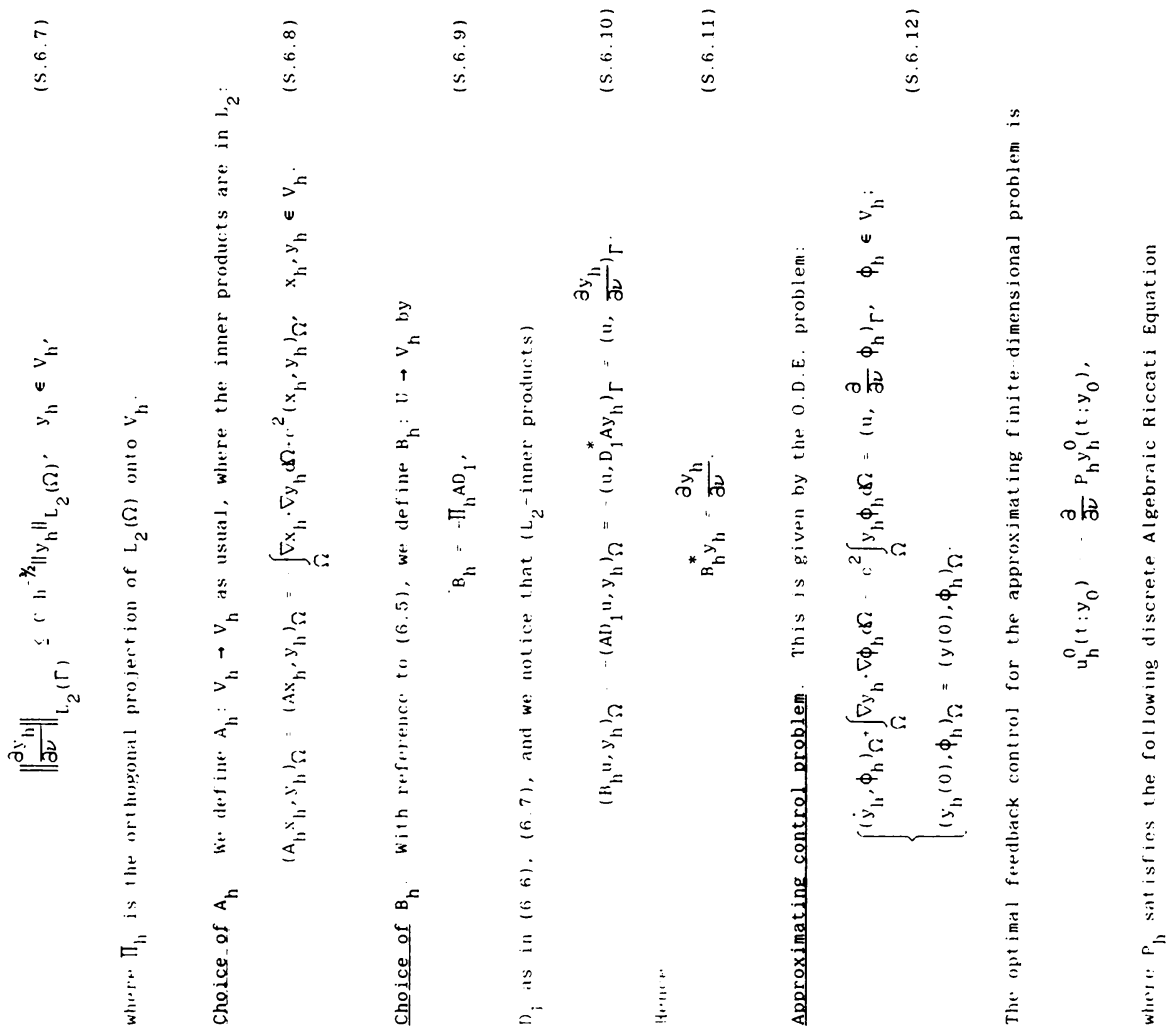



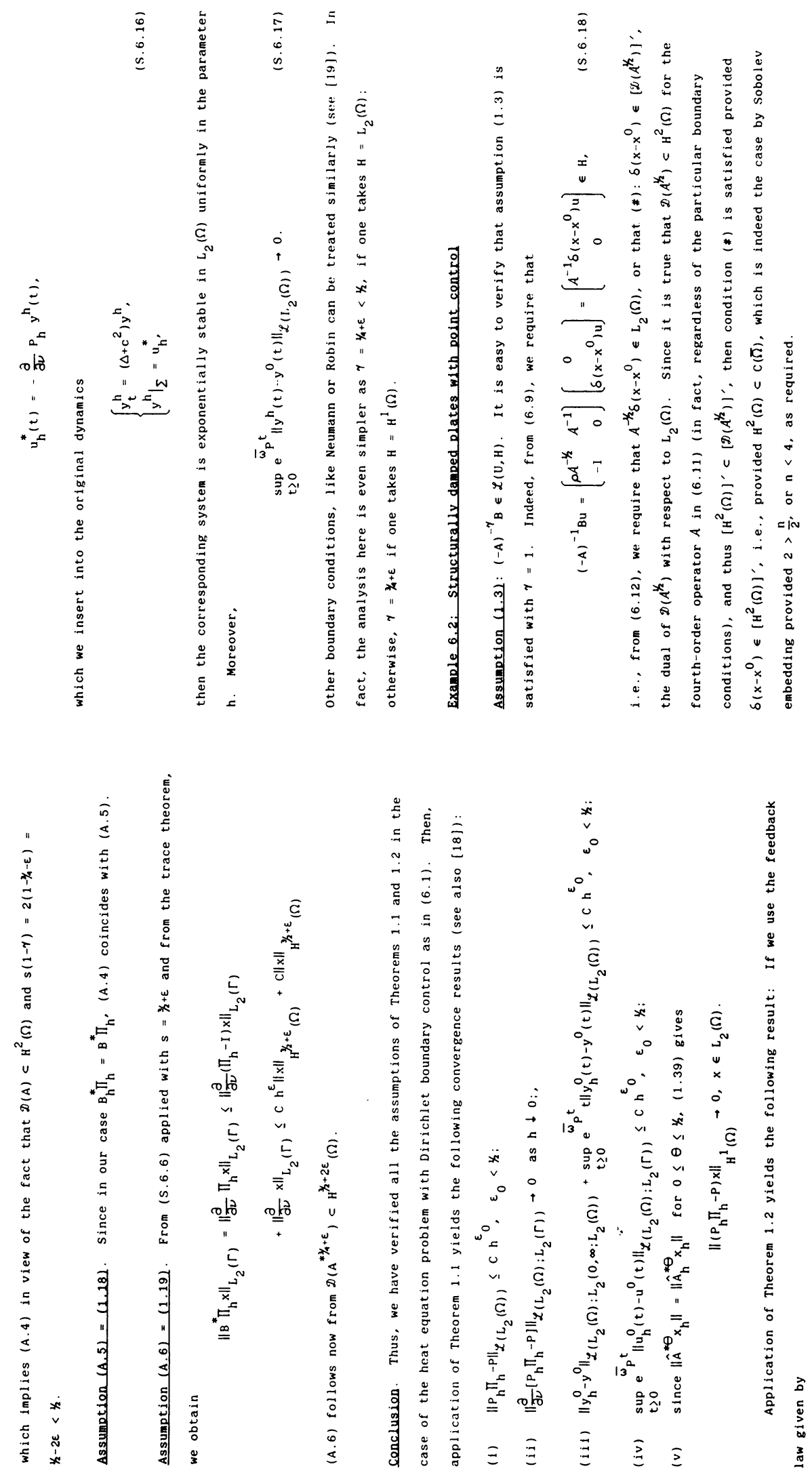

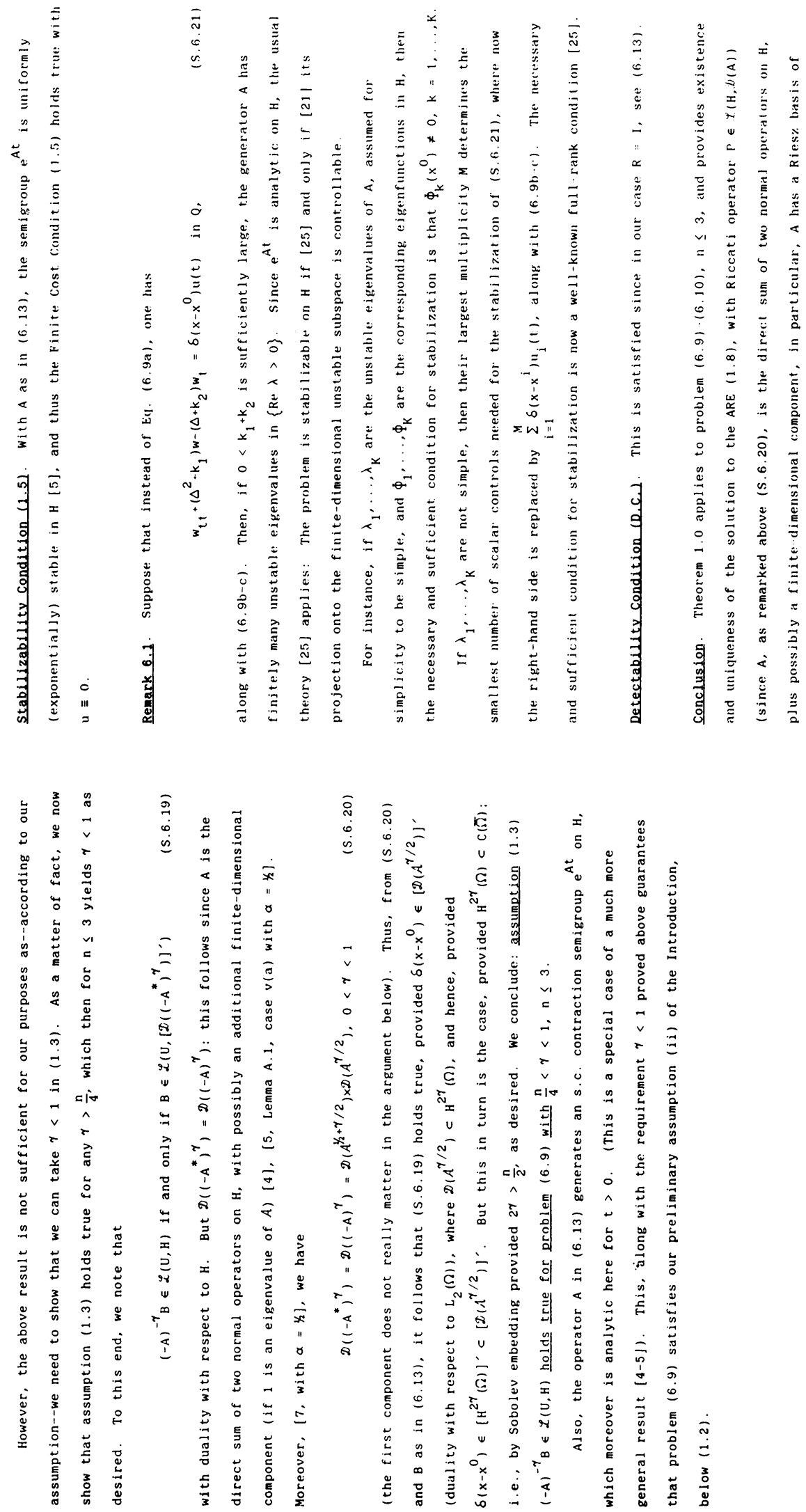

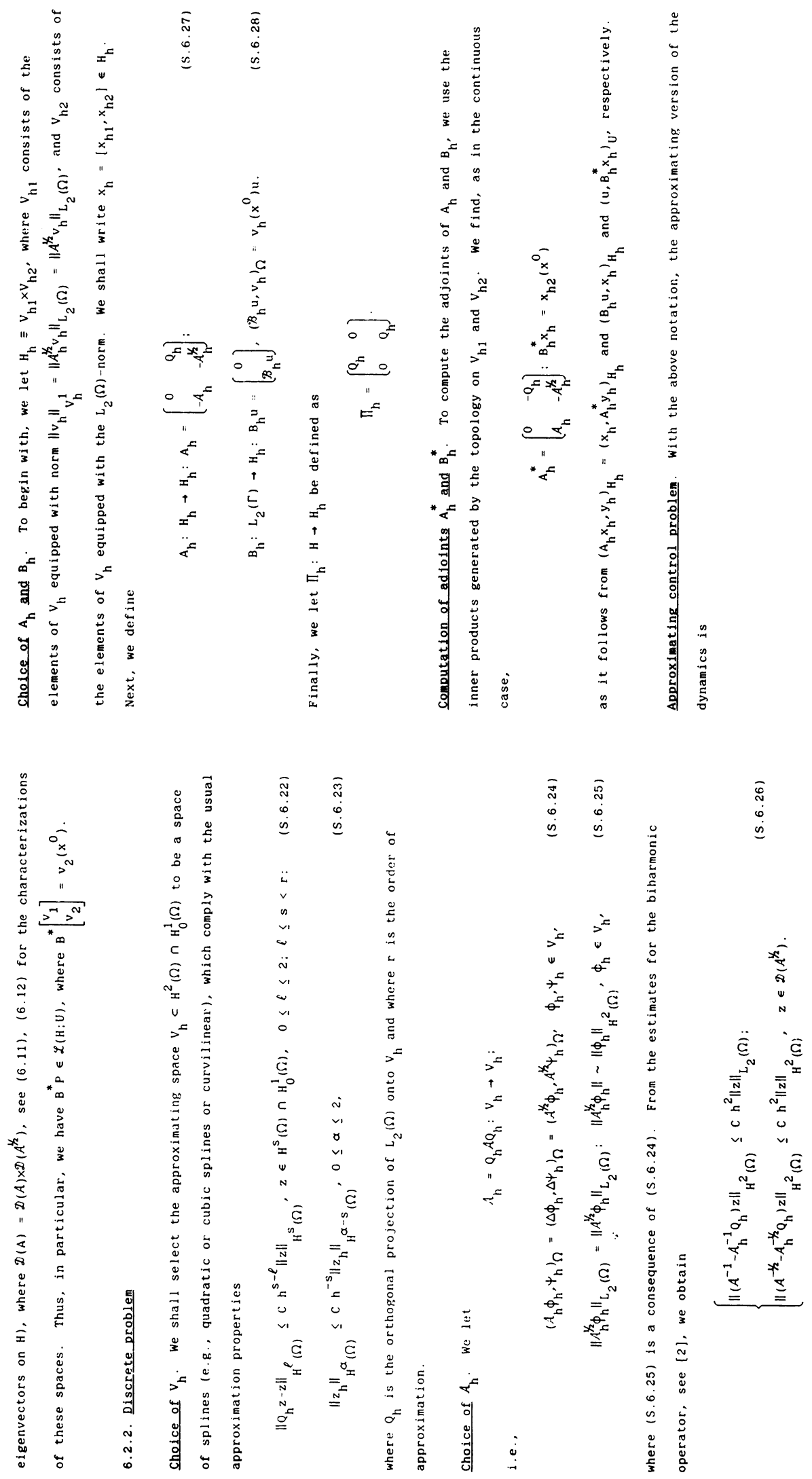

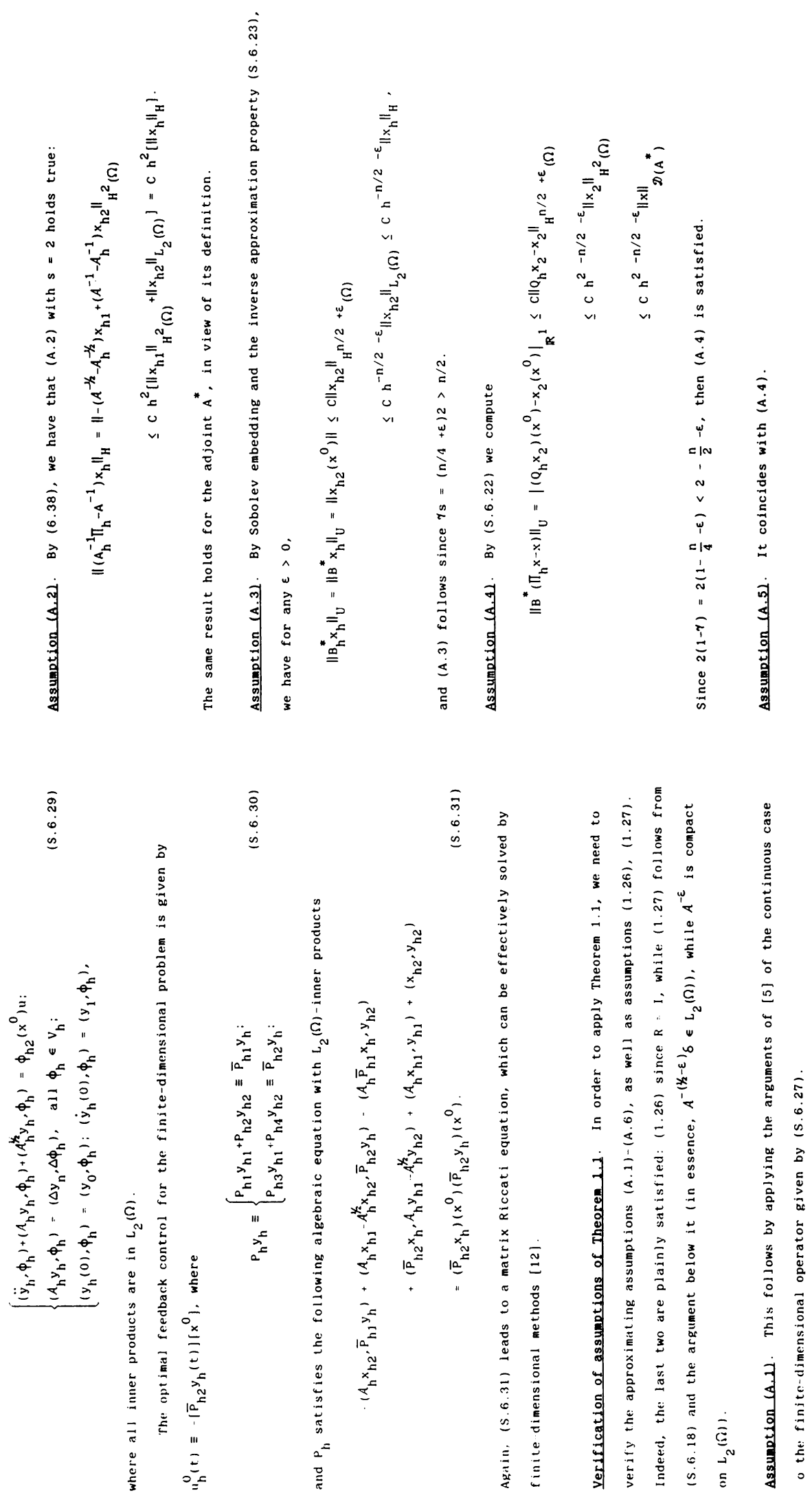

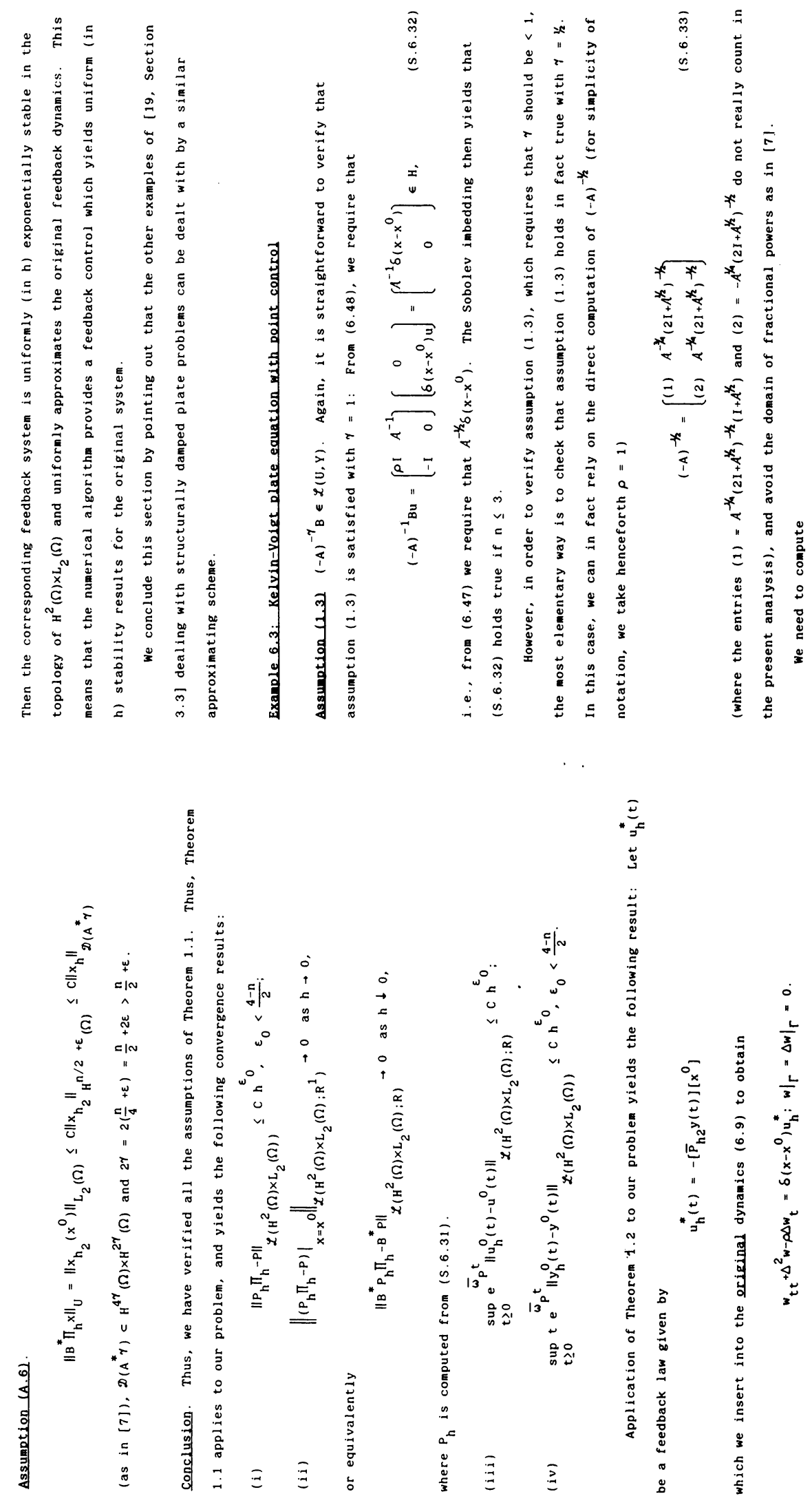


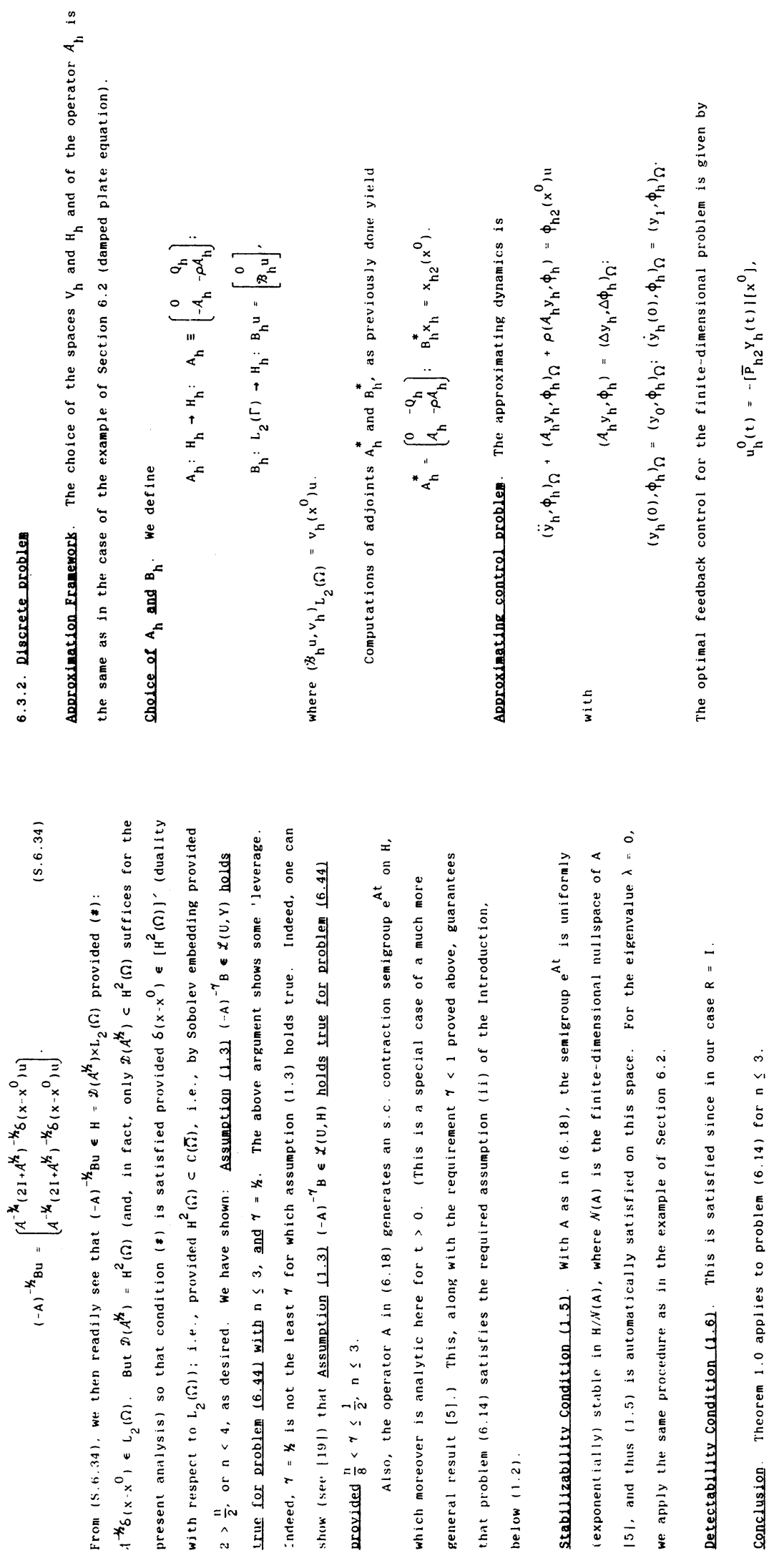



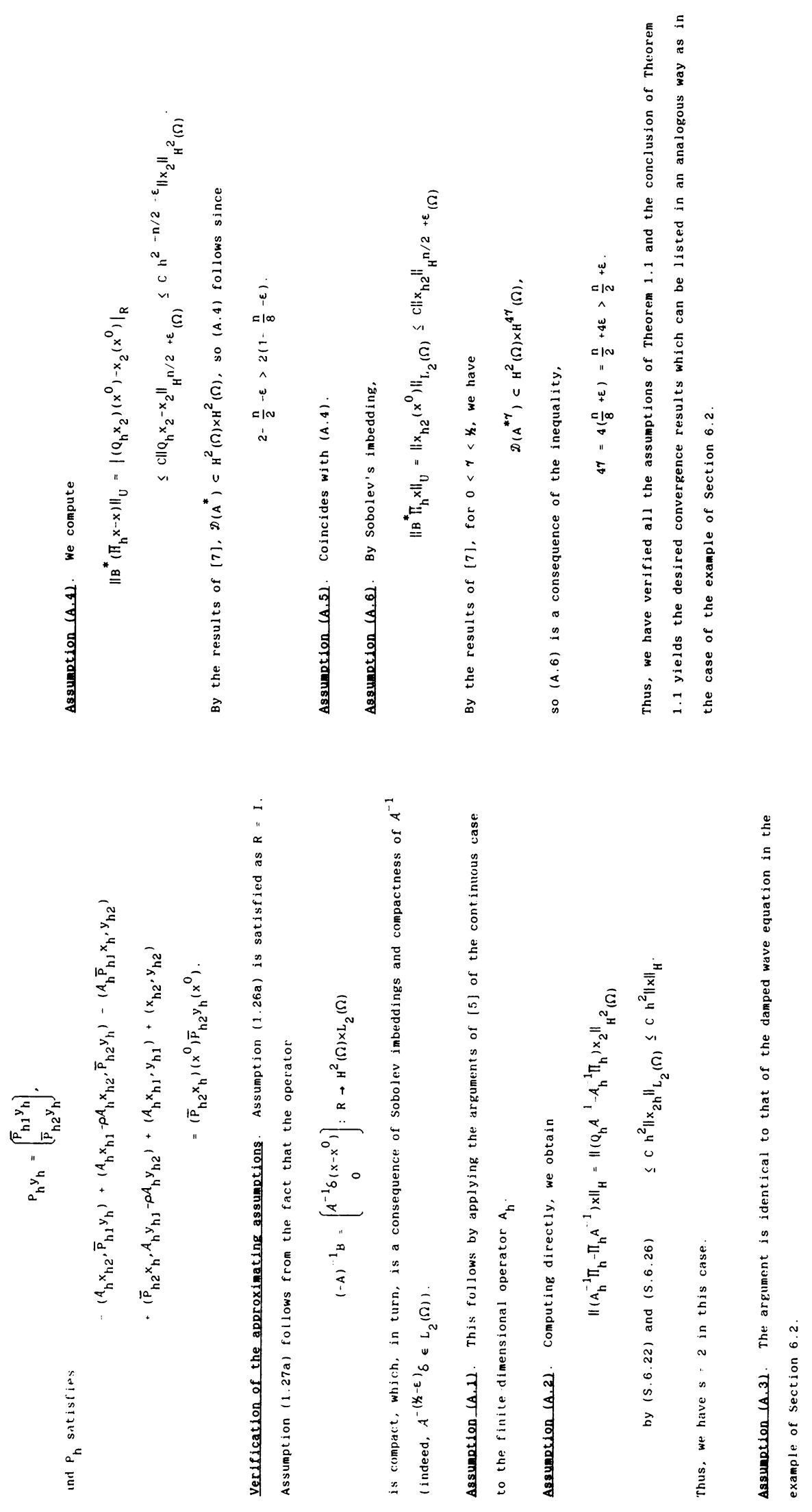